\title{
Complex-Distance Potential Theory and Hyperbolic Equations
}

\author{
Gerald Kaiser \\ Virginia Center for Signals and Waves \\ kaiser@wavelets.com・www.wavelets.com
}

June 15, 2021

\begin{abstract}
An extension of potential theory in $\mathbb{R}^{n}$ is obtained by continuing the Euclidean distance function holomorphically to $\mathbb{C}^{n}$. The resulting Newtonian potential is generated by an extended source distribution $\tilde{\delta}(\boldsymbol{z})$ in $\mathbb{C}^{n}$ whose restriction to $\mathbb{R}^{n}$ is the point source $\delta(\boldsymbol{x})$. This provides a possible model for extended particles in physics. In $\mathbb{C}^{n+1}$, interpreted as complex spacetime, $\tilde{\delta}$ acts as a propagator generating solutions of the wave equation from their initial values. This gives a new connection between elliptic and hyperbolic equations that does not assume analyticity of the Cauchy data. Generalized to Clifford analysis, it induces a similar connection between solutions of elliptic and hyperbolic Dirac equations. There is a natural application to the time-dependent, inhomogeneous Dirac and Maxwell equations, and the 'electromagnetic wavelets' introduced previously are an example.
\end{abstract}

\section{Motivation and Preliminaries}

Most fundamental theories of physics are based on the concept of potentials and fields generated by point sources, which presupposes that objects or "particles" can, in principle, be localized within arbitrarily small regions of space and/or time. This is a vast extrapolation from empirical evidence, and it should perhaps not come as a surprise if such theories experience some fundmental difficulties. In Newtonian mechanics, the problem of $N$ point "bodies" interacting through gravitation has, in general, no solution due to the possibility of collisions. This becomes a serious difficulty for $N \geq 3$, where the set of initial conditions leading to collisions is nontrivial [AM78, Chapter 10]. In classical electrodynamics, difficulties arise where the field produced by a point charge unavoidably acts back on the same charge, leading to infinite selfenergies and run-away particle trajectories [J99]. A way out of this dilemma was proposed by Wheeler and Feynman WF45, WF49, but their action-at-a distance theory, apart from being highly counter-intuitive [F64, p. 28-8], has resisted quantization and is not generally regarded as being a fundamental description of Nature. In quantum electrodynamics and other quantum field theories, point particles cause divergences which necessitate infinite "renormalization" procedures, a subject of some contraversy [C99]. String theory P98 does, in fact, not need infinite renormalization because its basic objects (strings) are extended in space rather than mathematical points. This is one of the reasons it is regarded with great hope as a possibility for unifying physical theories. However, a full development of (super)string physics is rather difficult and not expected to near completion for many years.

The ideas developed here began many years ago, motivated in part by the hope that an extension of physics to complex spacetime, justified at the foundational level, might give a way to circumvent the problems associated with point sources by applying residue methods. After some years of study and research this led to papers [K77, K78, K80, K87 and books [K90, K94] whose main thrust has been to develop a direct physical interpretation of the complex spacetime as an extended phase space, with the imaginary spacetime parameters carrying directional information while the real spacetime parameters describe (approximate!) localization. A major stumbling block in this program has been the construction of extended sources, since it seemed that they tend to spoil the holomorphy of the theory globally rather than just locally. Here we propose a natural solution to this problem. It turns out that although holomorphy enters the theory at the 
level of the fundamental potential or Green's function and this indeed gives a canonical extension of general fields to complex spacetime through convolution, these fields need not be holomorphic anywhere due to the specific structure of the extended sources.

We begin in Section 2 by extending the Euclidean distance $r(\boldsymbol{x})$ to a function $\gamma(\boldsymbol{x}+i \boldsymbol{y})$ holomorphic in a domain $\mathcal{O}_{n} \subset \mathbb{C}^{n}$. This leads to a natural coordinate system in $\mathbb{R}^{n}$ which will play an important role in the sequel: the oblate spheroidal coordinates adapted to $\boldsymbol{y} \neq \mathbf{0}$.

In Sections 3 and 1 we use $\gamma(\boldsymbol{z})$ to extend the Newtonian potential $\phi(\boldsymbol{x})$ (fundamental solution of the Laplacian in $\mathbb{R}^{n}$ ) to $\mathcal{O}_{n}$. The resulting holomorphic potential $\phi(\boldsymbol{z})$ has a source distribution $\tilde{\delta}(\boldsymbol{z})$ in $\mathbb{C}^{n}$ which, although nowhere holomorphic, is nevertheless a canonical extension to $\mathbb{C}^{n}$ of the point source $\delta(\boldsymbol{x})$ in $\mathbb{R}^{n}$. For even $n \geq 4, \tilde{\delta}(\boldsymbol{x}+i \boldsymbol{y})$ with fixed $\boldsymbol{y} \neq \mathbf{0}$ is supported in $\boldsymbol{x}$ on the sphere $\mathcal{B}(\boldsymbol{y})$ of codimension 2 and radius $|\boldsymbol{y}|$, centered at the origin and lying in the hyperplane orthogonal to $\boldsymbol{y}$. For all other values of $n$, it is supported in $\boldsymbol{x}$ on a membrane whose boundary is $\mathcal{B}(\boldsymbol{y})$. The membrane is determined by a choice of branch cut in $\gamma$, and in the simplest case it is a disk $E_{0}(\boldsymbol{y})$.

In Section 5 we compute the sources $\tilde{\delta}(\boldsymbol{x}+i \boldsymbol{y})$ explicitly as distributions in $\mathbb{R}^{3}$ and $\mathbb{R}^{4}$ supported, respectively, in $E_{0}(\boldsymbol{y})$ and $\mathcal{B}(\boldsymbol{y})$. This is especially interesting in the physical case of $\mathbb{R}^{3}$, where $\tilde{\delta}(\boldsymbol{x}+i \boldsymbol{y})$ consists of a uniform line charge on the rim of the disk $E_{0}(\boldsymbol{y})$ accompanied by a simple layer (with zero net charge) and a double layer distributed on the interior. Hence the original unit "charge" carried by $\delta(\boldsymbol{x})$ is now spread over a circle of radius $|\boldsymbol{y}|$, and a variable polarization density is induced on the disk swept out by "blowing up" the point source from the origin to its rim. That so much natural structure results from a simple analytic continuation is remarkable.

In Section 6 we show how to include time in this framework. Since we already have a holomorphic extension $\phi(\boldsymbol{x}, s+i t)$ of the fundamental solution $\phi(\boldsymbol{x}, s)$ of the Laplacian in $\mathbb{R}^{n+1}$, it is natural to interpret $t$ as a time parameter and look for a connection with the wave operator $\Delta_{\boldsymbol{x}}-\partial_{t}^{2}$, which is the "analytic continuation" to $\mathbb{R}^{n, 1}$ of the Laplacian $\Delta_{\boldsymbol{x}}+\partial_{s}^{2}$ in $\mathbb{R}^{n+1}$, now interpreted as Euclidean spacetime. We show that $\tilde{\delta}(\boldsymbol{x}, s+i t)$ acts as a propagator, generating solutions of the initial-value problem for the wave equation in $\mathbb{R}^{n, 1}$ from Cauchy data at $t=0$. This gives a connection between the Laplace equation and the wave equation without assuming analyticity of the initial data, because the propagator $\tilde{\delta}(\boldsymbol{x}, s+i t)$ is not holomorphic in $s+i t$.

In Section 7 we extend our method to Clifford analysis, where the Laplacian and the wave operator are replaced by their "square roots," the elliptic and hyperbolic Dirac operators. The time-dependent Maxwell equations are a natural application of the ensuing formalism.

The idea of extending harmonic functions to obtain solutions of the wave equation has been studied by Garabedian G64 and, in a more general context, by Ryan R90, R90a, R96a. However, the methods employed in these references depend critically on the assumption that the boundary data for the harmonic functions is holomorphic. As emphasized above, our method is free of this restriction.

\section{Complex Distance and Spheroidal Coordinates}

For $n \geq 2$, let

$$
\boldsymbol{z}=\boldsymbol{x}+i \boldsymbol{y} \in \mathbb{C}^{n}, \quad|\boldsymbol{x}|=r,|\boldsymbol{y}|=a .
$$

Define the complex length of $z$ as

$$
\gamma(\boldsymbol{z}) \equiv \sqrt{\boldsymbol{z}^{2}}=\sqrt{\boldsymbol{x}^{2}-\boldsymbol{y}^{2}+2 i \boldsymbol{x} \cdot \boldsymbol{y}}=\sqrt{r^{2}-a^{2}+2 i \boldsymbol{x} \cdot \boldsymbol{y}} \equiv p+i q .
$$

The complex distance between any two points $\boldsymbol{z}_{1}, \boldsymbol{z}_{2} \in \mathbb{C}^{n}$ is then defined as $\gamma\left(\boldsymbol{z}_{1}-\boldsymbol{z}_{2}\right)$. To complete this definition, a branch of the complex root must be chosen. The branch points form the null cone

$$
\mathcal{N}=\{\boldsymbol{z} \mid \gamma(\boldsymbol{z})=0\}=\left\{\boldsymbol{x}+i \boldsymbol{y} \mid \boldsymbol{x}^{2}=\boldsymbol{y}^{2} \text { and } \boldsymbol{x} \cdot \boldsymbol{y}=0\right\},
$$

a manifold of real dimension $2 n-2$ in $\mathbb{C}^{n} \approx \mathbb{R}^{2 n}$. In the context of the wave equation, $\mathcal{N}$ will be seen to be related to the light cone. We will be interested in the restriction of $\mathcal{N}$ to a fixed nonzero $\boldsymbol{y} \in \mathbb{R}^{n}$, i.e.,

$$
\mathcal{B} \equiv \mathcal{B}(\boldsymbol{y})=\left\{\boldsymbol{x} \in \mathbb{R}^{n} \mid r=a, \boldsymbol{x} \cdot \boldsymbol{y}=0\right\} .
$$


$\mathcal{B}$ is the sphere of dimension $n-2$ in $\mathbb{R}^{n}$ obtained by intersecting the sphere $r=a$ with the hyperplane orthogonal to $\boldsymbol{y}$. We call $\mathcal{B}(\boldsymbol{y})$ the branching sphere with axis vector $\boldsymbol{y}$.

We now concentrate on $\gamma(\boldsymbol{x}+i \boldsymbol{y})$ as a function of $\boldsymbol{x}$, regarding $\boldsymbol{y}$ as a fixed nonzero vector. In order to make this function single-valued, it is necessary to introduce a branch cut in $\mathbb{R}^{n}$. To see how this can be done, fix any $\boldsymbol{x} \in \mathcal{B}$. In the plane determined by the two orthogonal vectors $\boldsymbol{x}$ and $\boldsymbol{y}$, draw a circle of radius $\varepsilon \leq a$ centered at $\boldsymbol{x}$. Each time we go around this circle, $\gamma$ changes sign. In order to obtain a single-valued function for $\gamma$, it is therefore necessary to cut every such circle. Furthermore, this must be done subject to the requirement that $\gamma$ must be an extension to $\mathbb{C}^{n}$ of the usual length function in $\mathbb{R}^{n}$, i.e.,

$$
\boldsymbol{y} \rightarrow \mathbf{0} \Rightarrow \gamma(\boldsymbol{x}+i \boldsymbol{y}) \rightarrow r(\boldsymbol{x}) \geq 0 .
$$

The simplest cut (but certinainly not the only one) is obtained by requiring that

$$
p \equiv \operatorname{Re} \gamma \geq 0,
$$

which means that each of the above circles is cut at the point

$$
\boldsymbol{x}_{C}=(a-\varepsilon) \hat{\boldsymbol{x}}, \quad \hat{\boldsymbol{x}} \equiv \boldsymbol{x} / r .
$$

The totality of such points, as $\boldsymbol{x}$ varies over $\mathcal{B}$ and $0<\varepsilon \leq a$, together with $\mathcal{B}$ itself $(\varepsilon=0)$, forms the set

$$
E_{0} \equiv E_{0}(\boldsymbol{y})=\left\{\boldsymbol{x} \in \mathbb{R}^{n} \mid r \leq a, \boldsymbol{x} \cdot \boldsymbol{y}=0\right\},
$$

which is the disk of dimension $n-1$ in $\mathbb{R}^{n}$ obtained by intersecting the ball $r \leq a$ with the hyperplane through the origin orthogonal to $\boldsymbol{y}$. The boundary or rim of $E_{0}$ is $\mathcal{B}$ :

$$
\partial E_{0}=\mathcal{B}
$$

(For $n=3, E_{0}$ is indeed the disk of radius $a$ orthogonal to $\boldsymbol{y}$ and $\mathcal{B}$ is its rim.) In the context of holomorphic potential theory, $E_{0}$ or $\mathcal{B}$, depending on whether $n$ is odd or even, will represent the support of a source distribution. As $\boldsymbol{y} \rightarrow \mathbf{0}$, both sets contract to the origin, the support of the usual point source.

We now derive the basic properties of the real and imaginary parts $p, q$ of $\gamma$, which will be important in our study of the holomorphic potentials. From (2) it follows that

$$
p^{2}-q^{2}=r^{2}-a^{2}, \quad p q=\boldsymbol{x} \cdot \boldsymbol{y} \equiv a \zeta,
$$

where $\zeta$ is the projection of $\boldsymbol{x}$ onto $\boldsymbol{y} \neq \mathbf{0}$. Define the cylindrical coordinate $\rho$ by

$$
\rho^{2}=r^{2}-\zeta^{2}=a^{2}+p^{2}-q^{2}-\frac{p^{2} q^{2}}{a^{2}}=\frac{\left(a^{2}+p^{2}\right)\left(a^{2}-q^{2}\right)}{a^{2}} .
$$

This shows that the imaginary part of $\gamma$ is bounded by $a=|\boldsymbol{y}|$ :

$$
-a \leq q \leq a .
$$

We will use $(\rho, \zeta)$ as part of a cylindrical coordinate system in $\mathbb{R}^{n}$ with $\boldsymbol{y}$ as its " $z$-axis." Equations (8) and (9) show that the surface $E_{p}=\{\boldsymbol{x} \mid p=$ constant $\}$ with a given value of $p>0$ is an oblate spheroid given by

$$
E_{p}: \quad \frac{\rho^{2}}{a^{2}+p^{2}}+\frac{\zeta^{2}}{p^{2}}=1
$$

Similarly, the level surface $\left\{\boldsymbol{x} \mid q^{2}=\right.$ constant $\}$ with a given value of $0<q^{2}<a^{2}$ is a hyperboloid of one sheet given by

$$
H_{q}: \quad \frac{\rho^{2}}{a^{2}-q^{2}}-\frac{\zeta^{2}}{q^{2}}=1
$$


Note that we have so far avoided the surfaces with $p=0$ and $q=0, \pm a$. These give degenerate forms of $E_{p}$ and $H_{q}$. In fact, as $p \rightarrow+0$, the oblate spheroid $E_{p}$ contracts to the disk (7):

$$
p \rightarrow 0 \Rightarrow E_{p} \rightarrow\{\boldsymbol{x} \mid \zeta=0, \rho \leq a\}=E_{0} .
$$

More precisely, since the interior of $E_{0}$ is covered twice, we will distinguish between its front and back sides:

$$
E_{0}=E_{0}^{+} \cup E_{0}^{-} \cup \mathcal{B}
$$

where

$$
E_{0}^{ \pm}=\{\boldsymbol{x} \mid \zeta= \pm 0, \rho<a\}=\{\boldsymbol{x} \mid p=0, \pm q>0\}
$$

are the interiors of the front and back sides of $E_{0}$. Although $E_{0}^{+}$and $E_{0}^{-}$coincide as sets, the distinction between them will be very important for the following reason. It can be easily seen that

$$
\boldsymbol{x} \in E_{0}^{ \pm} \Rightarrow q= \pm \sqrt{a^{2}-r^{2}}= \pm \sqrt{a^{2}-\rho^{2}} .
$$

This shows that while $p$ is continuous across $E_{0}, q$ has a spherical jump discontinuity there. In the context of holomorphic potential theory, $E_{0}^{+}$and $E_{0}^{-}$will be seen to form the two sides of a double layer.

The degenerate values of $q$ are $q=0, \pm a$. As $q \rightarrow \pm a$, the semi-hyperboloids $H_{q}^{ \pm}$with $\pm q>0$ collapse to the positive and negative $\zeta$-axis, respectively. As $q \rightarrow 0, H_{q}$ collapses to the set $H_{0}=\{\boldsymbol{x} \mid \zeta=0, \rho \geq a\}$ which, like $E_{0}$, is covered twice, once by the limit of each semi-hyperboloid $H_{q}^{ \pm}$as $q \rightarrow \pm 0$. But in this case we do not distinguish between the two copies because $\gamma$ is continuous across $H_{0}$.

As mentioned above, the parameters $\zeta$ and $\rho$ are part of a cylindrical coordinate system in $\mathbb{R}^{n}$. Note that the intersection of $E_{p}$ and $H_{q}$ is

$$
S_{\gamma}=E_{p} \cap H_{q}=\left\{\boldsymbol{x} \in \mathbb{R}^{n} \mid a \zeta=p q, a \rho=\sqrt{a^{2}+p^{2}} \sqrt{a^{2}-q^{2}}\right\} .
$$

Here and henceforth, we denote the pair of real variables $(p, q)$ by the single complex variable $\gamma=p+i q$ for convenience. If $q \neq \pm a$, then $S_{\gamma}$ is a sphere of dimension $n-2$ and radius $\rho$. The variable point on $S_{\gamma}$ may be represented as

$$
\boldsymbol{x}=\rho \boldsymbol{\sigma}+\zeta \hat{\boldsymbol{y}} \in S_{\gamma},
$$

where $\boldsymbol{\sigma}$ runs over the unit sphere of dimension $n-2$ in the hyperplane orthogonal to $\boldsymbol{y}$. In particular, $\gamma=0$ gives $\zeta=0$ and $\rho=a$, so that

$$
S_{0}=\left\{a \boldsymbol{\sigma} \mid \boldsymbol{\sigma} \in S^{n-2}\right\}=\mathcal{B}(\boldsymbol{y}) .
$$

If $n=2, S_{\gamma}$ consists of just two points. If $n=3, S_{\gamma}$ is a circle. Thus, for fixed $\boldsymbol{y} \neq \mathbf{0}$, a complete set of cylindrical coordinates in $\mathbb{R}^{n}$ is given by $(\rho, \zeta, \boldsymbol{\sigma})$. Since $\zeta$ and $\rho$ are functions of $(p, q)$, we may equivalently use the coordinates

$$
\boldsymbol{x}=(p, q, \boldsymbol{\sigma}) \equiv(\gamma, \boldsymbol{\sigma})
$$

This is the oblate spheroidal coordinate system in $\mathbb{R}^{n}$, long known as a useful tool in the theory of special functions [T96. It is remarkable that this system is so intimately related to the holomorphic extension of the distance function.

Now let $\nabla$ be the gradient with respect to $\boldsymbol{x}$ with $\boldsymbol{y}$ held constant, and let $\Delta=\nabla^{2}$ be the Laplacian in $\mathbb{R}^{n}$. Then

$$
\gamma^{2}=\boldsymbol{z}^{2} \Rightarrow \gamma \nabla \gamma=\boldsymbol{z}, \quad \text { hence } \quad(\nabla \gamma)^{2} \equiv \nabla \gamma \cdot \nabla \gamma=1 .
$$

Taking the divergence of (19) gives

$$
\nabla \gamma \cdot \nabla \gamma+\gamma \Delta \gamma=n, \Rightarrow \Delta \gamma=\frac{n-1}{\gamma} .
$$


Now

$$
\nabla \gamma=\frac{\boldsymbol{z}}{\gamma}=\frac{\bar{\gamma} \boldsymbol{z}}{\bar{\gamma} \gamma} \Rightarrow \nabla p=\frac{p \boldsymbol{x}+q \boldsymbol{y}}{\bar{\gamma} \gamma}, \quad \nabla q=\frac{p \boldsymbol{y}-q \boldsymbol{x}}{\bar{\gamma} \gamma},
$$

therefore

$$
\begin{gathered}
\nabla \gamma \cdot \nabla \gamma=1 \Rightarrow(\nabla p)^{2}-(\nabla q)^{2}=1, \quad \nabla p \cdot \nabla q=0 \\
\nabla \bar{\gamma} \cdot \nabla \gamma=\frac{|\boldsymbol{z}|^{2}}{\bar{\gamma} \gamma} \Rightarrow(\nabla p)^{2}+(\nabla q)^{2}=\frac{r^{2}+a^{2}}{\bar{\gamma} \gamma}
\end{gathered}
$$

which gives

$$
(\nabla p)^{2}=\frac{a^{2}+p^{2}}{\bar{\gamma} \gamma}, \quad(\nabla q)^{2}=\frac{a^{2}-q^{2}}{\bar{\gamma} \gamma} .
$$

Finally, the real and imaginary parts of (20) give

$$
\Delta p=\frac{n-1}{\bar{\gamma} \gamma} p, \quad \Delta q=-\frac{n-1}{\bar{\gamma} \gamma} q .
$$

We will need to compute volume integrals in the oblate spheroidal coordinates. Note that the area of the unit sphere $S^{n-1} \subset \mathbb{R}^{n}$ is $\mathrm{CH} 62$

$$
\omega_{n}=\frac{2 \pi^{n / 2}}{\Gamma(n / 2)}, \quad n \geq 2 .
$$

Let $d \boldsymbol{\sigma}$ denote the surface measure on the unit sphere $S^{n-2}$ in the hyperplane orthogonal to $\boldsymbol{y} \neq \mathbf{0}$, normalized so that

$$
\int_{S^{n-2}} d \boldsymbol{\sigma}=1
$$

Then for fixed $\boldsymbol{y}$, the volume measure $d \boldsymbol{x}$ in $\mathbb{R}^{n}$ is given in cylindrical coordinates by

$$
d \boldsymbol{x}=\omega_{n-1} \rho^{n-2} d \rho d \zeta d \boldsymbol{\sigma} .
$$

By (8) and (9),

$$
\begin{aligned}
\rho^{n-2} d \rho d \zeta & =\frac{\rho^{n-3}}{2} d\left[\rho^{2}\right] d \zeta=\frac{\rho^{n-3}}{2 a^{3}} d\left[\left(a^{2}+p^{2}\right)\left(a^{2}-q^{2}\right)\right] d[p q] \\
& =a^{-1} \rho^{n-3}(p d p-q d q)(p d q+q d p)=a^{-1} \rho^{n-3}\left(p^{2}+q^{2}\right) d p d q,
\end{aligned}
$$

where $d p d q$ denotes the antisymmetric exterior product of differential forms (see [GS64, for example). Therefore the volume measure in the oblate spheroidal coordinates is given by

$$
d \boldsymbol{x}=\frac{\omega_{n-1}}{a} \rho^{n-3}\left(p^{2}+q^{2}\right) d p d q d \boldsymbol{\sigma}=\frac{\omega_{n-1}}{a} \rho^{n-3} \bar{\gamma} \gamma d p d q d \boldsymbol{\sigma} .
$$

\section{Holomorphic Potentials and Their Sources}

For simplicity, we now assume that $n \geq 3$. The case $n=2$ is similar but requires some special attention and will be described elsewhere. Consider the fundamental solution $\phi(\boldsymbol{x})$ of Laplace's equation defined by

$$
\Delta \phi(\boldsymbol{x})=\delta(\boldsymbol{x}), \quad \lim _{r \rightarrow \infty} \phi(\boldsymbol{x})=0 .
$$

It is given uniquely by $\mathrm{CH} 62$

$$
\phi(\boldsymbol{x})=\frac{1}{\omega_{n}} \frac{r^{2-n}}{2-n}, \quad \boldsymbol{x} \in \mathbb{R}^{n}, n \geq 3 .
$$


For $n=3, \phi(\boldsymbol{x})=-1 / 4 \pi r$ is the Newton-Coulomb potential with unit mass or charge. Define the holomorphic potential in $\mathbb{C}^{n}$ by

$$
\phi(\boldsymbol{z})=\frac{1}{\omega_{n}} \frac{\gamma^{2-n}}{2-n}, \quad \boldsymbol{z} \in \mathbb{C}^{n}, n \geq 3 .
$$

For odd $n, \phi(\boldsymbol{z})$ inherits the branch cut of $\gamma(\boldsymbol{z})$. For even $n$, the only singularities occur on $\mathcal{B}$, where $\gamma=0$. Thus $\gamma(\boldsymbol{z})$ and $\phi(\boldsymbol{z})$ are analytic continuations of $r(\boldsymbol{x})$ and $\phi(\boldsymbol{x})$ to the domains

$$
\begin{aligned}
& \mathcal{O}_{n}=\left\{\boldsymbol{z} \in \mathbb{C}^{n} \mid p>0\right\}=\left\{\boldsymbol{x}+i \boldsymbol{y} \mid \boldsymbol{x} \notin E_{0}(\boldsymbol{y})\right\}, \quad \text { odd } n \geq 3 \\
& \mathcal{O}_{n}=\left\{\boldsymbol{z} \in \mathbb{C}^{n} \mid \gamma \neq 0\right\}=\{\boldsymbol{x}+i \boldsymbol{y} \mid \boldsymbol{x} \notin \mathcal{B}(\boldsymbol{y})\}, \quad \text { even } n \geq 4 \text {. }
\end{aligned}
$$

Proposition 1 For fixed $\boldsymbol{y}, \phi(\boldsymbol{x}+i \boldsymbol{y})$ is harmonic with respect to $\boldsymbol{x}$ when $\boldsymbol{x}+i \boldsymbol{y} \in \mathcal{O}_{n}$.

Proof: By (32), we have

$$
\omega_{n} \nabla \phi(\boldsymbol{z})=\gamma^{1-n} \nabla \gamma
$$

Thus by (20),

$$
\omega_{n} \Delta \phi(\boldsymbol{z})=(1-n) \gamma^{-n}+\gamma^{1-n} \Delta \gamma=0 .
$$

Our objective is to compute the source distribution of $\phi(\boldsymbol{z})$, which we define formally by analogy with (30) as

$$
\tilde{\delta}(\boldsymbol{z})=\Delta \phi(\boldsymbol{z}), \quad \boldsymbol{z} \in \mathbb{C}^{n} .
$$

This will be shown to be a generalized function GS64 of $\boldsymbol{x}$ for any fixed $\boldsymbol{y}$, meaning that given any sufficienlty smooth "test" function $f(\boldsymbol{x})$, the integral

$$
\langle\tilde{\delta}, f\rangle=\int_{\mathbb{R}^{n}} \tilde{\delta}(\boldsymbol{x}+i \boldsymbol{y}) f(\boldsymbol{x}) d \boldsymbol{x}
$$

defines a bounded linear functional of $f$. (Generalized functions are more commonly known as distributions [265. We use the former term here in order to avoid confusion with the term "source distribution.")

By Proposition 1, $\tilde{\delta}(\boldsymbol{x}+i \boldsymbol{y})$ is supported on $\boldsymbol{x} \in \mathcal{B}$ for even $n \geq 4$, and on $\boldsymbol{x} \in E_{0}$ otherwise. In any case, it has compact support in the variable $\boldsymbol{x}$, and as $\boldsymbol{y} \rightarrow \mathbf{0}$, this support contracts to the origin. We will show that

$$
\boldsymbol{y} \rightarrow \mathbf{0} \Rightarrow\langle\tilde{\delta}, f\rangle \rightarrow f(\mathbf{0}), \text { hence } \tilde{\delta}(\boldsymbol{x}+i \boldsymbol{y}) \rightarrow \delta(\boldsymbol{x}) .
$$

To compute the generalized function $\tilde{\delta}(\boldsymbol{z})$, we first define the regularized potential:

$$
\phi_{\varepsilon}(\boldsymbol{z})=\theta(p-\varepsilon) \phi(\boldsymbol{z}), \quad \varepsilon>0, \quad \text { where } \quad \theta(\xi)= \begin{cases}1 & \text { if } \xi>0 \\ 0 & \text { if } \xi<0\end{cases}
$$

is the (Heaviside) unit step function. The regularization $(35)$ eliminates the singularities on $E_{0}$ and $\mathcal{B}$, replacing them by a discontinuity in $\phi_{\varepsilon}$ across the spheroid $E_{\varepsilon}$. Thus, while the source of the singular potential $\phi(\boldsymbol{z})$ is concentrated on $E_{0}$ or $\mathcal{B}$, that of the regularized potential $\phi_{\varepsilon}(\boldsymbol{z})$ is concentrated on $E_{\varepsilon}$. The advantage gained is that while $\phi(\boldsymbol{x}+i \boldsymbol{y})$ is infinite for $\boldsymbol{x} \in \mathcal{B} \subset E_{0}, \phi_{\varepsilon}(\boldsymbol{x}+i \boldsymbol{y})$ remains bounded in a neighborhood of $E_{\varepsilon}$.

Now $\phi_{\varepsilon}(\boldsymbol{z})$ vanishes in the interior of $E_{\varepsilon}$ but is identical to $\phi(\boldsymbol{z})$ in the exterior. Therefore its source distribution, defined as a generalized function by

$$
\tilde{\delta}_{\varepsilon}(\boldsymbol{z}) \equiv \Delta \phi_{\varepsilon}(\boldsymbol{z})
$$


represents an equivalent source distribution on $\boldsymbol{x} \in E_{\varepsilon}$ whose potential field simulates that of $\tilde{\delta}(\boldsymbol{z})$ in the exterior of $E_{\varepsilon}$ but vanishes in the interior. We call $\tilde{\delta}_{\varepsilon}(\boldsymbol{z})$ the regularized source distribution, and will define the singular source distribution $\tilde{\delta}(\boldsymbol{z})$ as the limit of $\tilde{\delta}_{\varepsilon}(\boldsymbol{z})$ in the sense of generalized functions, for any fixed $\boldsymbol{y} \in \mathbb{R}^{n}$. That is,

$$
\langle\tilde{\delta}, f\rangle \equiv \lim _{\varepsilon \rightarrow 0^{+}}\left\langle\tilde{\delta}_{\varepsilon}, f\right\rangle
$$

for every test function $f(\boldsymbol{x})$ in $\mathbb{R}^{n}$. Using $\theta^{\prime}(\xi)=\delta(\xi)$, (35) gives

$$
\nabla \phi_{\varepsilon}=\delta(p-\varepsilon) \phi \nabla p+\theta(p-\varepsilon) \nabla \phi,
$$

hence

$$
\Delta \phi_{\varepsilon}=\delta^{\prime}(p-\varepsilon) \phi(\nabla p)^{2}+2 \delta(p-\varepsilon) \nabla \phi \cdot \nabla p+\delta(p-\varepsilon) \phi \Delta p+\theta(p-\varepsilon) \Delta \phi .
$$

By Proposition 1, $\Delta \phi$ vanishes for $p \geq \varepsilon / 2$, so the last term in (38) vanishes identically. The remaining terms show that $\delta_{\varepsilon}(\boldsymbol{x}+i \boldsymbol{y})$ is indeed supported on $E_{\varepsilon}$ as expected. Inserting

$$
\nabla \phi=\phi^{\prime}(\gamma) \nabla \gamma=\frac{\gamma^{1-n} \nabla \gamma}{\omega_{n}}
$$

into (38) and using (22)-25), we have

$$
\tilde{\delta}_{\varepsilon}(\boldsymbol{z})=\left[\delta^{\prime}(p-\varepsilon) \phi+2 \delta(p-\varepsilon) \phi^{\prime}\right] \frac{a^{2}+p^{2}}{\bar{\gamma} \gamma}+\delta(p-\varepsilon) \phi \frac{n-1}{\bar{\gamma} \gamma} p .
$$

This expression represents a generalized function of $\boldsymbol{x}$. To make sense of it we must apply it to a test function $f(\boldsymbol{x})$ in $\mathbb{R}^{n}$, assumed to be sufficiently smooth. (That is, $f$ is assumed to possess all derivatives which the ensuing computation requires it to possess. As will be seen, the required degree of smoothness increases with $n$.) For a given value of $\boldsymbol{y} \in \mathbb{R}^{n}, \tilde{\delta}_{\varepsilon}$ acts on $f$ as in (34),

$$
\left\langle\tilde{\delta}_{\varepsilon}, f\right\rangle=\int_{\mathbb{R}^{n}} \tilde{\delta}_{\varepsilon}(\boldsymbol{x}+i \boldsymbol{y}) f(\boldsymbol{x}) d \boldsymbol{x} .
$$

Using the oblate spheroidal coordinates (18), let us write

$$
f(\boldsymbol{x})=f(\rho \boldsymbol{\sigma}+\zeta \hat{\boldsymbol{y}})=f^{\sharp}(p, q, \boldsymbol{\sigma}) \equiv f^{\sharp}(\gamma, \boldsymbol{\sigma}),
$$

where the two expressions on the right are obtained by substituting (8) and (9) for $\zeta$ and $\rho$ in terms of $(p, q)$. Let

$$
\bar{f}^{\sharp}(\gamma)=\int_{S^{n-2}} f^{\sharp}(\gamma, \boldsymbol{\sigma}) d \boldsymbol{\sigma}=\int_{S^{n-2}} f(\rho \boldsymbol{\sigma}+\zeta \hat{\boldsymbol{y}}) d \boldsymbol{\sigma} \equiv \bar{f}(\rho, \zeta) .
$$

The notations $f^{\sharp}(\gamma, \boldsymbol{\sigma})$ and $\bar{f}^{\sharp}(\gamma) \equiv \bar{f}^{\sharp}(p, q)$ are used for convenience and are not meant to imply analyticity in $\gamma$. Because of the normalization (27), $\bar{f}^{\sharp}(\gamma)$ and $\bar{f}(\rho, \zeta)$ are the means of $f^{\sharp}(\gamma, \boldsymbol{\sigma})$ and $f(\rho \boldsymbol{\sigma}+\zeta \hat{\boldsymbol{y}})$ over the sphere $S_{\gamma}=E_{p} \cap H_{q}=\{\boldsymbol{x}:|\boldsymbol{x}-\zeta \boldsymbol{\sigma}|=\rho\}$. Using the expression (29) for $d \boldsymbol{x}$, (40) becomes

$$
\left\langle\tilde{\delta}_{\varepsilon}, f\right\rangle=\frac{\omega_{n-1}}{a} \int_{0}^{\infty} d p \int_{-a}^{a} d q \rho^{n-3} \bar{\gamma} \gamma \tilde{\delta}_{\varepsilon}(\gamma) \bar{f}^{\sharp}(\gamma),
$$

where we have used the fact that $\tilde{\delta}_{\varepsilon}(\boldsymbol{z})$ in (39) is independent of $\boldsymbol{\sigma}$ to write it as $\tilde{\delta}_{\varepsilon}(p, q) \equiv \tilde{\delta}_{\varepsilon}(\gamma)$. By (9),

$$
\left(a^{2}+p^{2}\right) \partial_{p} \rho^{n-3}=(n-3) p \rho^{n-3} .
$$

Hence the first term in (39) gives, upon integrating by parts over $p$,

$$
\begin{gathered}
\int_{0}^{\infty} d p \int_{-a}^{a} d q\left(a^{2}+p^{2}\right) \rho^{n-3} \delta^{\prime}(p-\varepsilon) \phi(\gamma) \bar{f}^{\sharp}(\gamma) \\
=-\int_{-a}^{a} d q \rho^{n-3}\left[(n-3) \varepsilon \phi \bar{f}^{\sharp}+2 \varepsilon \phi \bar{f}^{\sharp}+\left(a^{2}+p^{2}\right)\left(\phi^{\prime} \bar{f}^{\sharp}+\phi \bar{f}_{p}^{\sharp}\right)\right] \\
=-\int_{-a}^{a} d q \rho^{n-3}\left[(n-1) \varepsilon \phi \bar{f}^{\sharp}+\left(a^{2}+\varepsilon^{2}\right)\left(\phi^{\prime} \bar{f}^{\sharp}+\phi \bar{f}_{p}^{\sharp}\right)\right],
\end{gathered}
$$


where $\bar{f}_{p}^{\sharp}=\partial_{p} \bar{f}^{\sharp}(p, q)$ and the integrand is to be evaluated at $p=\varepsilon$. Inserting the other terms in (39) and simplifying, we obtain

$$
\left\langle\tilde{\delta}_{\varepsilon}, f\right\rangle=\frac{a^{2}+\varepsilon^{2}}{a} \omega_{n-1} \int_{-a}^{a} d q \rho^{n-3}\left[\phi^{\prime} \bar{f}^{\sharp}-\phi \bar{f}_{p}^{\sharp}\right] .
$$

Proposition 2 For all $n \geq 3$, the regularized source distribution $\tilde{\delta}_{\varepsilon}(\boldsymbol{x}+i \boldsymbol{y})$ is supported on the oblate spheroid $\boldsymbol{x} \in E_{\varepsilon}$, and its action on a test function $f(\boldsymbol{x})=f^{\sharp}(\gamma, \boldsymbol{\sigma})$ is given by

$$
\left\langle\tilde{\delta}_{\varepsilon}, f\right\rangle=I_{\varepsilon}\left(\bar{f}^{\sharp}\right),
$$

where $I_{\varepsilon}$ is the linear functional defined by

$$
I_{\varepsilon}\left(\bar{f}^{\sharp}\right)=\frac{\left(a^{2}+\varepsilon^{2}\right)^{\nu+1}}{a^{n-2} A_{n}} \int_{-a}^{a} \frac{F^{\sharp}(\varepsilon+i q)}{(\varepsilon+i q)^{n-1}} d q, \quad \nu=\frac{n-3}{2},
$$

with

$$
A_{n}=\frac{\omega_{n}}{\omega_{n-1}}, \quad F^{\sharp}(\gamma)=\left(a^{2}-q^{2}\right)^{\nu}\left[\bar{f}^{\sharp}(\gamma)+\frac{\gamma \bar{f}_{p}^{\sharp}(\gamma)}{n-2}\right] .
$$

Proof: This follows immediately from (42), using

$$
\rho^{2}=\frac{\left(a^{2}+\varepsilon^{2}\right)\left(a^{2}-q^{2}\right)}{a^{2}} .
$$

Let us verify that as $\boldsymbol{y} \rightarrow \mathbf{0}$ and the source disk shrinks to a point, the source of the singular potential $\phi$ contracts to the usual point source. Letting $q=a \xi$ in (45), we have

$$
F^{\sharp}(\gamma)=a^{n-3}\left(1-\xi^{2}\right)^{\nu}\left[\bar{f}^{\sharp}(\gamma)+\frac{\gamma \bar{f}_{p}^{\sharp}(\gamma)}{n-2}\right], \quad \text { where } \quad \gamma=\varepsilon+i a \xi .
$$

Therefore

$$
I_{\varepsilon}\left(\bar{f}^{\sharp}\right)=\frac{\left(a^{2}+\varepsilon^{2}\right)^{\frac{n-1}{2}}}{A_{n}} \int_{-1}^{1} \frac{\left(1-\xi^{2}\right)^{\nu}}{(\varepsilon+i a \xi)^{n-1}}\left[\bar{f}^{\sharp}(\gamma)+\frac{\gamma \bar{f}_{p}^{\sharp}(\gamma)}{n-2}\right] d \xi
$$

and

$$
\lim _{a \rightarrow 0} I_{\varepsilon}\left(\bar{f}^{\sharp}\right)=\left[\bar{f}^{\sharp}(\varepsilon)+\frac{\varepsilon \bar{f}_{p}^{\sharp}(\varepsilon)}{n-2}\right] \frac{K_{n}}{A_{n}},
$$

where

$$
K_{n}=\int_{-1}^{1}\left(1-\xi^{2}\right)^{\nu} d \xi=B\left(\frac{1}{2}, \frac{n-1}{2}\right)=\frac{\sqrt{\pi} \Gamma\left(\frac{n-1}{2}\right)}{\Gamma\left(\frac{n}{2}\right)}=\frac{\omega_{n}}{\omega_{n-1}}=A_{n}
$$

Thus by (43),

$$
\lim _{a \rightarrow 0}\left\langle\tilde{\delta}_{\varepsilon}, f\right\rangle=\bar{f}^{\sharp}(\varepsilon)+\frac{\varepsilon \bar{f}_{p}^{\sharp}(\varepsilon)}{n-2} .
$$

Now let $\varepsilon \rightarrow 0$, and note that since $E_{\varepsilon}$ contracts to the origin,

$$
\lim _{\varepsilon \rightarrow 0} \bar{f}^{\sharp}(\varepsilon)=\lim _{\varepsilon \rightarrow 0} \int_{S^{n-2}} f^{\sharp}(\varepsilon, \boldsymbol{\sigma}) d \boldsymbol{\sigma}=f(\mathbf{0}), \text { hence } \lim _{\varepsilon \rightarrow 0} \lim _{a \rightarrow 0}\left\langle\tilde{\delta}_{\varepsilon}, f\right\rangle=f(\mathbf{0}) .
$$

If we assume that the two limits can be exchanged (as will be verified later) and use the definition (37) of $\tilde{\delta}$, this states that $\lim _{a \rightarrow 0}\langle\tilde{\delta}, f\rangle=f(\mathbf{0})$, giving the following important result.

Theorem 1 The singular source distribution $\tilde{\delta}(\boldsymbol{z})$ is an extension of the usual point-source distribution in $\mathbb{R}^{n}$, in the sense that

$$
\boldsymbol{y} \rightarrow \mathbf{0} \Rightarrow \tilde{\delta}(\boldsymbol{x}+i \boldsymbol{y}) \rightarrow \delta(\boldsymbol{x})
$$




\section{Singular Source Distributions}

We are ready at last compute the singular source distributions. By (37) and (43), they given by the limit

$$
\langle\tilde{\delta}, f\rangle=\lim _{\varepsilon \rightarrow 0} I_{\varepsilon}\left(\bar{f}^{\sharp}\right) .
$$

However, we cannot simply let $\varepsilon=0$ in the expression (44) for $I_{\varepsilon}\left(\bar{f}^{\sharp}\right)$, since the resulting integral diverges. Recall the decomposition of $E_{0}$ given in Equation (14):

$$
E_{0}=E_{0}^{+} \cup E_{0}^{-} \cup \mathcal{B}
$$

where $E_{0}^{ \pm}$are the interiors of the front and back sides of $E_{0}$ and $\mathcal{B}$ is its rim, the branching sphere. This decomposition recognizes the nature of $E_{0}$ as a limit of closed ellipsoids. Although the two open disks $E_{0}^{ \pm}$ look identical to a continuous function, they look distinct to a generalized function like $\tilde{\delta}$ which is singular across $E_{0}$. Furthermore, the oblate spheroidal coordinates are an ideal tool for resolving this decomposition since $q>0$ on $E_{0}^{+}, q<0$ on $E_{0}^{-}$and $q=0$ on $\mathcal{B}$. We will compute $\langle\tilde{\delta}, f\rangle$ by decomposing the integral $I_{\varepsilon}\left(\bar{f}^{\sharp}\right)$ in a way similar to (48) and then taking the limit $\varepsilon \rightarrow 0$. The integral over $E_{0}^{+} \cup E_{0}^{-}$gives a sum of single and double layer distibutions of dimension $n-1$ over the interior of the disk $E_{0}$, while the integral over $\mathcal{B}$ gives a boundary distribution of dimension $n-2$ over $\mathcal{B}$. All these distributions are well-defined, giving a finite expression for $\langle\tilde{\delta}, f\rangle$.

The divergence of $I_{0}\left(\bar{f}^{\sharp}\right)$ is therefore caused by the attempt to represent the boundary distribution on $\mathcal{B}$ as part of an integral of dimension $n-1$ over $E_{0}$, and the above regularization simply amounts to recognizing this fact.

To regularize the integral

$$
I_{\varepsilon}\left(\bar{f}^{\sharp}\right)=\frac{\left(a^{2}+\varepsilon^{2}\right)^{\nu+1}}{a^{n-2} A_{n}} \int_{-a}^{a} \frac{F^{\sharp}(\varepsilon+i q)}{(\varepsilon+i q)^{n-1}} d q,
$$

define the Taylor coefficients

$$
T_{m}(\varepsilon)=\left.\frac{1}{m !} \partial_{q}^{m} F^{\sharp}(\varepsilon+i q)\right|_{q=0}, \quad T_{m} \equiv T_{m}(0)
$$

and the Taylor polynomials approximating $F^{\sharp}(\varepsilon+i q)$ and $F^{\sharp}(i q)$ to order $q^{n-2}$,

$$
F_{n-2}^{\sharp}(\varepsilon, q)=\sum_{m=0}^{n-2} q^{m} T_{m}(\varepsilon), \quad F_{n-2}^{\sharp}(q)=\sum_{m=0}^{n-2} q^{m} T_{m} .
$$

We rewrite the integral in (49) as

$$
\int_{-a}^{a} \frac{F^{\sharp}(\varepsilon+i q)}{(\varepsilon+i q)^{n-1}} d q=\int_{-a}^{a} \frac{F^{\sharp}(\varepsilon+i q)-F_{n-2}^{\sharp}(\varepsilon, q)}{(\varepsilon+i q)^{n-1}} d q+\sum_{m=0}^{n-2} i^{-m} T_{m}(\varepsilon) \lambda_{k}^{m}(\varepsilon),
$$

where

$$
\lambda_{k}^{m}(\varepsilon)=\int_{-a}^{a} \frac{i^{m} q^{m} d q}{(\varepsilon+i q)^{k}}, \quad 0 \leq m<k .
$$

If $f(\boldsymbol{x})$ is differentiable to order $n-2$, so is $F^{\sharp}(\varepsilon+i q) \equiv F^{\sharp}(\varepsilon, q)$. Then

$$
F^{\sharp}(\varepsilon+i q)-F_{n-2}^{\sharp}(\varepsilon, q)=O\left(q^{n-1}\right)
$$

and the integral on the right-hand side of (52) has a finite limit as $\varepsilon \rightarrow 0$. It therefore remains to compute the limit of the sum. We begin by finding

$$
\lambda_{1}^{0}(\varepsilon)=\int_{-a}^{a} \frac{d q}{\varepsilon+i q}=2 \varepsilon \int_{0}^{a} \frac{d q}{\varepsilon^{2}+q^{2}}=2 \tan ^{-1} \frac{a}{\varepsilon}=\pi-2 \tan ^{-1} \frac{\varepsilon}{a} \equiv \alpha(\varepsilon) .
$$


All the other integrals (53) can be computed from the recursion relations

$$
\begin{aligned}
\lambda_{k+1}^{0}(\varepsilon) & =-\frac{1}{k} \partial_{\varepsilon} \lambda_{k}^{0}(\varepsilon)=\frac{(-1)^{k}}{k !} \partial_{\varepsilon}^{k} \alpha(\varepsilon) \\
\lambda_{k}^{m+1}(\varepsilon) & =i^{m} \int_{-a}^{a} \frac{(\varepsilon+i q-\varepsilon) q^{m} d q}{(\varepsilon+i q)^{k}}=\lambda_{k-1}^{m}(\varepsilon)-\varepsilon \lambda_{k}^{m}(\varepsilon) .
\end{aligned}
$$

We are interested in the limit $\varepsilon \rightarrow 0$, where these relations imply

$$
\begin{gathered}
\lambda_{k+1} \equiv \lambda_{k+1}^{0}(0)=\frac{(-1)^{k}}{k !} \partial_{\varepsilon}^{k} \alpha(0) \equiv(-1)^{k} L_{k} \\
\lambda_{k}^{m}(0)=\lambda_{k-m}^{0}(0)=\lambda_{k-m}, \quad 0 \leq m<k .
\end{gathered}
$$

The Taylor coefficients $L_{k}$ of $\alpha(\varepsilon)$ are obtained from the expansion

$$
\alpha(\varepsilon)=\pi-2 \tan ^{-1} \frac{\varepsilon}{a}=\pi+2 \sum_{l=1}^{\infty} \frac{(-1)^{l}}{2 l-1} \frac{\varepsilon^{2 l-1}}{a^{2 l-1}} .
$$

Thus

$$
\lambda_{1}=\pi, \quad \lambda_{2 l}=\frac{2(-1)^{l+1}}{(2 l-1) a^{2 l-1}}, \quad \lambda_{2 l+1}=0, \quad l \geq 1 .
$$

By (56), this gives finite values for all the coefficients $\lambda_{k}^{m}(0)$. Note that the original expression (53) diverges if we set $\varepsilon=0$. The finiteness of the limits depends on delicate cancellations of contributions from positive and negative values of $q$ when $\varepsilon>0$, just as happens in Cauchy's principal value integral. In fact, (54) shows that the present regularization reduces to the principal value integral when $k=1$.

Using (52), we can now compute the limit $\varepsilon \rightarrow 0$ in (47):

$$
\begin{aligned}
\langle\tilde{\delta}, f\rangle & =V_{n}(f)+\frac{a}{A_{n}} \sum_{m=0}^{n-2} i^{-m} T_{m} \lambda_{n-m-1}, \\
V_{n}(f) & \equiv \frac{i^{1-n} a}{A_{n}} \int_{-a}^{a} \frac{F^{\sharp}(i q)-F_{n-2}^{\sharp}(q)}{q^{n-1}} d q .
\end{aligned}
$$

$V_{n}(f)$ will be shown to be a bounded linear functional of $f$, and this establishes $\tilde{\delta}$ as a well-defined generalized function.

Since $\bar{f}^{\sharp}(0)$ is the mean of $f$ over $\mathcal{B}$, the terms in the sum in (59) represent means of $f$ and its derivatives over $\mathcal{B}$. On the other hand, (60) represents an integral of $f$ and its normal derivative over the interior $E_{0}^{+} \cup E_{0}^{-}$, since the boundary terms have already been subtracted in the form of $F_{n-2}^{\sharp}(q)$. Thus, (59) is the promised decomposition of the source distribution corresponding to (48).

Equation (59) can be greatly simplified because of certain symmetries satisfied by $\bar{f}^{\sharp}(i q)$. We claim that

$$
\bar{f}^{\sharp}(-i q)=\bar{f}^{\sharp}(i q), \quad \bar{f}_{p}^{\sharp}(-i q)=-\bar{f}_{p}^{\sharp}(i q), \quad \bar{f}_{q}^{\sharp}(-i q)=-\bar{f}_{q}^{\sharp}(i q) .
$$

To see this, note that the coordinates $\gamma= \pm i q$ denote the same point of $E_{0}$, regarded as belonging to $E_{0}^{ \pm}$. Since the test function $f(\boldsymbol{x})$ is continuous across $E_{0}$, so is its integral $\bar{f}^{\sharp}(i q)$, and this proves the first relation. On the other hand, $p$ increases in the $\pm \zeta$ direction on $E_{0}^{ \pm}$, which proves the second relation. Finally, the third relation follows from the first by differentiation.

By (61), the function

$$
F^{\sharp}(i q)=\left(a^{2}-q^{2}\right)^{\nu}\left[\bar{f}^{\sharp}(i q)+i \frac{q \bar{f}_{p}^{\sharp}(i q)}{n-2}\right]
$$


is even, so its odd Taylor coefficients vanish:

$$
m \text { is odd } \Rightarrow T_{m}=0 .
$$

Furthermore, 60 shows that

$$
n \text { is even } \Rightarrow V_{n}(f)=0 \text {. }
$$

Considering the cases of odd and even $n$ separately and inserting the values of $\lambda_{k}$ from (58) gives the following result.

Theorem 2 The singular source distribution $\tilde{\delta}(\boldsymbol{z})$ is a bounded linear functional which acts on a test function $f \in C^{n-2}\left(\mathbb{R}^{n}\right)$ as follows. For even $n=2 k+2 \geq 4$,

$$
\langle\tilde{\delta}, f\rangle=\frac{\pi a}{A_{n}}(-1)^{k} T_{2 k},
$$

where $A_{n}$ and $T_{2 k}$ are given by (45) and (50). For odd $n=2 k+3 \geq 3$,

$$
\langle\tilde{\delta}, f\rangle=V_{n}(f)+\frac{2(-1)^{k}}{A_{n}} \sum_{l=0}^{k} \frac{a^{2 l-2 k} T_{2 l}}{2 k-2 l+1},
$$

where $V_{n}(f)$ is given by (60).

Proof: To prove (65), note first that $V_{n}(f)$ vanishes by (64). Furthermore, the sum in (59) reduces to a single term because $T_{m} \lambda_{n-m-1}=0$ unless $m$ is even, which implies that $n-m-1$ is odd; but the only nonvanishing coefficient $\lambda_{l}$ with odd $l$ is $\lambda_{1}=\pi$.

Equation (66) follows directly from (59), (58) and (63). To establish $\tilde{\delta}$ as a distribution, we must still prove that $V_{n}(f)$ is a bounded linear functional for odd $n$. Since the integrand in (60) is even when $n$ is odd, we have

$$
V_{n}(f)=\frac{2 i^{1-n} a}{A_{n}} \int_{0}^{a} \frac{F^{\sharp}(i q)-F_{n-2}^{\sharp}(q)}{q^{n-1}} d q .
$$

By Taylor's theorem,

$$
F^{\sharp}(i q)-F_{n-2}^{\sharp}(q)=\frac{1}{(n-2) !} \int_{0}^{q}(q-w)^{n-2}\left(F^{\sharp}\right)^{(n-1)}(i w) d w .
$$

Therefore

$$
\left|F^{\sharp}(i q)-F_{n-2}^{\sharp}(q)\right| \leq q^{n-1} M, \quad M=\frac{\max \left|\left(F^{\sharp}\right)^{(n-1)}(i w)\right|}{(n-2) !},
$$

and

$$
\left|V_{n}(f)\right| \leq 2 a^{2} M
$$

Since $M$ depends boundedly on $f$, this shows that $V_{n}(f)$ is a bounded linear functional as claimed.

Thus, for even $n \geq 4$, the integral over the interior of $E_{0}$ vanishes and the singular source is concentrated on $\mathcal{B}$. This is to be expected, since $\gamma^{2-n}$ has no branch cut and therefore the only singularity occurs on the boundary.

We can now derive a useful expression for the source distribution $\tilde{\delta}(\boldsymbol{x}+i \boldsymbol{y})$ in the cylindrical coordinates $(\rho, \zeta, \boldsymbol{\sigma})$ adapted to $\boldsymbol{y} \neq \mathbf{0}$. As a byproduct, it will be seen that for all $n \geq 3$, the test function $f(\boldsymbol{x})$ need only be in $C^{k}\left(\mathbb{R}^{n}\right)$ with $k=\left[\frac{n-1}{2}\right]$, instead of $C^{n-2}\left(\mathbb{R}^{n}\right)$ as assumed in Theorem 2 . 
Our first task is to rewrite the function $F^{\sharp}(i q)$ in (62) in terms of cylindrical coordinates. Recall that

$$
\begin{gathered}
\zeta=\frac{p q}{a}, \quad \rho=\frac{\sqrt{\left(a^{2}+p^{2}\right)\left(a^{2}-q^{2}\right)}}{a} \\
\boldsymbol{x}=\rho \boldsymbol{\sigma}+\zeta \hat{\boldsymbol{y}}, \quad \boldsymbol{\sigma} \in S^{n-2} \perp \boldsymbol{y}, \\
f(\rho \boldsymbol{\sigma}+\zeta \hat{\boldsymbol{y}})=f^{\sharp}(p, q, \boldsymbol{\sigma}) .
\end{gathered}
$$

Then the mean of $f$ on $S_{\gamma}=E_{p} \cap H_{q}$ becomes

$$
\bar{f}^{\sharp}(p, q)=\int_{S^{n-2}} h(\rho \boldsymbol{\sigma}+\zeta \hat{\boldsymbol{y}}) d \boldsymbol{\sigma} \equiv \bar{f}(\rho, \zeta),
$$

which is the mean of $f$ on the sphere $S_{\rho, \zeta}=\{\boldsymbol{x}:|\boldsymbol{x}-\zeta \hat{\boldsymbol{y}}|=\rho\}$.

It will be shown elsewhere $\mathrm{K} 00$ that the source distributions for odd $n$ can be derived from those for even $n$ by integrating over one of the coordinates. Specifically, we have:

Theorem 3 Let $n \geq 3$, and denote points in $\mathbb{C}^{n+1}$ by

$$
\mathbf{z}=\mathbf{x}+i \mathbf{y}=(\boldsymbol{z}, s+i t), \quad \boldsymbol{z} \in \mathbb{C}^{n} .
$$

For fixed $\mathbf{y} \neq \mathbf{0}$, the singular source distributions $\tilde{\delta}_{n}(\boldsymbol{x}+i \boldsymbol{y})$ in $\mathbb{R}^{n}$ and $\tilde{\delta}_{n+1}(\mathbf{x}+i \mathbf{y})$ in $\mathbb{R}^{n+1}$ are related by

$$
\tilde{\delta}_{n}(\boldsymbol{z})=\int_{-\infty}^{\infty} d s \tilde{\delta}_{n+1}(\boldsymbol{z}, s) .
$$

That is, for a test function $f(\boldsymbol{x})$ in $\mathbb{R}^{n}$ we have

$$
\left\langle\tilde{\delta}_{n}, f\right\rangle=\int_{-\infty}^{\infty} d s \int_{\mathbb{R}^{n}} d \boldsymbol{x} \tilde{\delta}_{n+1}(\boldsymbol{x}+i \boldsymbol{y}, s) f(\boldsymbol{x}) .
$$

For $\boldsymbol{y}=\mathbf{0}$, (70) follows from $\delta_{n+1}(\boldsymbol{x}, s)=\delta_{n}(\boldsymbol{x}) \delta(s)$. For $\boldsymbol{y} \neq \mathbf{0}$, this tensor product decomposition fails but Theorem 3 still holds. We therefore state the following theorem for the simpler case of even $n \geq 4$, though the transformation to cylindrical coordinates derived below will be used later in the explicit computation of the extended source in $\mathbb{R}^{3}$.

Theorem 4 For $n=2 k+2 \geq 4$, the source distribution $\tilde{\delta}$ is a bounded linear functional on test fucntions $f(\boldsymbol{x})$ in $C^{k}\left(\mathbb{R}^{n}\right)$ whose action is given in the cylindrical coordinates $(\rho, \zeta, \boldsymbol{\sigma})$ by

$$
\langle\tilde{\delta}, f\rangle=\left.\frac{a \sqrt{\pi}}{\Gamma\left(k+\frac{1}{2}\right)} D_{\rho}^{k} F(\rho)\right|_{\rho=a}
$$

where

$$
D_{\rho}=\frac{\partial}{\partial\left(\rho^{2}\right)}=\frac{1}{2 \rho} \frac{\partial}{\partial \rho}, \quad F(\rho)=\rho^{2 k-1}\left[\bar{f}(\rho, 0)+i \frac{a^{2}-\rho^{2}}{2 k a} \bar{f}_{\zeta}(\rho, 0)\right]
$$

Proof: By (67), we have

$$
\bar{f}_{p}^{\sharp}(p, q)=\frac{\rho p}{a^{2}+p^{2}} \bar{f}_{\rho}(\rho, \zeta)+\frac{q}{a} \bar{f}_{\zeta}(\rho, \zeta) .
$$

Since

$$
p=0 \Rightarrow q^{2}=a^{2}-\rho^{2},
$$


it follows from (62) that

$$
\begin{aligned}
F(i q) & =\left(a^{2}-q^{2}\right)^{\nu}\left[\bar{f}^{\sharp}(0, q)+i \frac{q \bar{f}_{p}^{\sharp}(0, q)}{n-2}\right] \\
& =\rho^{n-3}\left[\bar{f}(\rho, 0)+i \frac{q^{2}}{(n-2) a} \bar{f}_{\zeta}(\rho, 0)\right]=F(\rho) .
\end{aligned}
$$

Note that (75) makes sense only because $F(i q)$ is even and so does not depend on the sign of $q$, which cannot be recovered from $\rho$ when $\zeta=0$ since $\operatorname{sgn} q=\operatorname{sgn} \zeta$. With

$$
D_{q}=\frac{\partial}{\partial\left(q^{2}\right)}=\frac{1}{2 q} \frac{\partial}{\partial q}=-D_{\rho} \quad \text { when } \quad p=0,
$$

we have

$$
F(i q)=\sum_{m=0}^{\infty} T_{2 m} q^{2 m} \Rightarrow T_{2 m}=\left.\frac{1}{m !} D_{q}^{m} F(i q)\right|_{q=0}=\left.\frac{(-1)^{m}}{m !} D_{\rho}^{m} F(\rho)\right|_{\rho=a} .
$$

For $n=2 k+2 \geq 4$,

$$
\omega_{n}=\frac{2 \pi^{k+1}}{\Gamma(k+1)}=\frac{2 \pi^{k+1}}{k !}, \quad \omega_{n-1}=\frac{2 \pi^{k+\frac{1}{2}}}{\Gamma\left(k+\frac{1}{2}\right)}
$$

and (65) gives

$$
\langle\tilde{\delta}, f\rangle=\frac{\pi a \omega_{n-1}}{\omega_{n}}(-1)^{k} T_{2 k}=\left.\frac{a \sqrt{\pi}}{\Gamma\left(k+\frac{1}{2}\right)} D_{\rho}^{k} F(\rho)\right|_{\rho=a} .
$$

Now $F(\rho)$ already contains one derivative $\left(\bar{f}_{\zeta}\right)$ and $D_{\rho}^{k}$ computes $k$ more, hence it suffices to have $f \in$ $C^{k+1}\left(\mathbb{R}^{n}\right)$. But the highest derivative of $f$ occuring in $(72)$ is

$$
\frac{\rho^{2 k-1}}{(2 \rho)^{k}} \cdot i \frac{a^{2}-\rho^{2}}{2 k a} \partial_{\rho}^{k} \bar{f}_{\zeta}(\rho, 0)
$$

which vanishes at $\rho=a$. Hence the highest nonvanishing derivative is of order $k$, so it suffices for $\bar{f}(\rho, \zeta)$ to be $k$ times continuously differentiable as claimed, and so does $f(\boldsymbol{x})$.

An application of (71) now shows that for $n=2 k+1$, we need $f \in C^{k}\left(\mathbb{R}^{n}\right)$ in order for $\left\langle\tilde{\delta}_{n}, f\right\rangle$ to make sense. Thus for any $n \geq 3, f$ needs to be in $C^{k}\left(\mathbb{R}^{n}\right)$ with

$$
k=\left[\frac{n-1}{2}\right]= \begin{cases}\frac{n-2}{2}, & n \text { even } \\ \frac{n-1}{2}, & n \text { odd. }\end{cases}
$$

\section{Computation of Sources in $\mathbb{R}^{3}$ and $\mathbb{R}^{4}$}

We now compute the singular source distribution in $\mathbb{R}^{3}$ explicitly and interpret the result. Equation (62) becomes

$$
F^{\sharp}(i q)=\bar{f}^{\sharp}(i q)+i q \bar{f}_{p}^{\sharp}(i q),
$$

hence $T_{0}=\bar{f}^{\sharp}(0), T_{1}=0$, and (51) becomes

$$
F_{1}^{\sharp}(q)=\bar{f}^{\sharp}(0) .
$$

Equation (66) therefore gives

$$
\langle\tilde{\delta}, f\rangle=-a \int_{-a}^{a} \frac{\bar{f}^{\sharp}(i q)+i q \bar{f}_{p}^{\sharp}(i q)-\bar{f}^{\sharp}(0)}{q^{2}} d q+\bar{f}^{\sharp}(0) .
$$

Using the symmetry of the integrand, we obtain the following result. 
Proposition 3 The singular source distribution

$$
\tilde{\delta}(\boldsymbol{x}+i \boldsymbol{y})=-\Delta \frac{1}{4 \pi \gamma}, \quad \boldsymbol{x}+i \boldsymbol{y} \in \mathbb{C}^{3},
$$

is a bounded linear functional whose action on a test function $f(\boldsymbol{x})=f^{\sharp}(\gamma, \boldsymbol{\sigma})$ in $C^{1}\left(\mathbb{R}^{3}\right)$, is given in oblate spheroidal and cylindrical coordinates by

$$
\langle\tilde{\delta}, h\rangle=L_{0}+L_{1}+i L_{2}
$$

where

$$
\begin{aligned}
& L_{0}=\bar{f}^{\sharp}(0)=\bar{f}(a, 0) \\
& L_{1}=-a \int_{0}^{a} \frac{\bar{f}^{\sharp}(i q)-\bar{f}^{\sharp}(0)}{q^{2}} d q=-a \int_{0}^{a} \frac{\bar{f}(\rho, 0)-\bar{f}(a, 0)}{\left(a^{2}-\rho^{2}\right)^{3 / 2}} \rho d \rho \\
& L_{2}=-a \int_{0}^{a} \frac{\bar{f}_{p}^{\sharp}(i q)}{q} d q=-\int_{0}^{a} \frac{\bar{f}_{\zeta}(\rho, 0)}{\sqrt{a^{2}-\rho^{2}}} \rho d \rho .
\end{aligned}
$$

Proof: The action in oblate spheroidal coordinates follows immediately from (66). To obtain the action in cylindrical coordinates, use (74).

Note that since

$$
\lim _{a \rightarrow 0} \bar{f}(a, 0)=f(\mathbf{0}) \quad \text { and } \quad \lim _{a \rightarrow 0} L_{1}=\lim _{a \rightarrow 0} L_{2}=0
$$

(77) shows that

$$
\boldsymbol{y} \rightarrow \mathbf{0} \Rightarrow \tilde{\delta}(\boldsymbol{x}+i \boldsymbol{y}) \rightarrow \delta(\boldsymbol{x}) .
$$

This was already seen in Theorem 1 for all $n \geq 3$, but that proof was less rigorous because it depended on the assumption that the order of the limits $a \rightarrow 0$ and $\varepsilon \rightarrow 0$ can be exchanged.

We now state some other interesting properties of the expression (77) which will help interpret its three terms.

Proposition 4 The monopole and dipole moments of $\tilde{\delta}(\boldsymbol{x}+i \boldsymbol{y})$ in $\mathbb{R}^{3}$ are

$$
Q \equiv \int_{\mathbb{R}^{3}} \tilde{\delta}(\boldsymbol{x}+i \boldsymbol{y}) d \boldsymbol{x}=1, \quad \boldsymbol{P} \equiv \int_{\mathbb{R}^{3}} \boldsymbol{x} \tilde{\delta}(\boldsymbol{x}+i \boldsymbol{y}) d \boldsymbol{x}=-i \boldsymbol{y} .
$$

Given a point source with general complex coordinates $\boldsymbol{z}_{S}=\boldsymbol{x}_{S}+i \boldsymbol{y}_{S} \in \mathbb{C}^{3}$, the centroid of its charge distribution is

$$
\boldsymbol{C}\left(\boldsymbol{z}_{S}\right) \equiv \int_{\mathbb{R}^{3}} \boldsymbol{x} \tilde{\delta}\left(\boldsymbol{x}-\boldsymbol{z}_{S}\right) d \boldsymbol{x}=\boldsymbol{z}_{S} .
$$

Proof: To compute $Q$, apply Proposition 3 with $f(\boldsymbol{x}) \equiv 1$. To find $\boldsymbol{P}$, apply it to the vector-valued test function

$$
\boldsymbol{f}(\boldsymbol{x})=\boldsymbol{x}=\rho \boldsymbol{\sigma}+\zeta \hat{\boldsymbol{y}}
$$

Finally,

$$
\boldsymbol{C}\left(\boldsymbol{z}_{S}\right)=\boldsymbol{x}_{S} \int_{\mathbb{R}^{3}} \tilde{\delta}\left(\boldsymbol{x}-\boldsymbol{x}_{S}-i \boldsymbol{y}_{S}\right) d \boldsymbol{x}+\int_{\mathbb{R}^{3}}\left(\boldsymbol{x}-\boldsymbol{x}_{S}\right) \tilde{\delta}\left(\boldsymbol{x}-\boldsymbol{x}_{S}-i \boldsymbol{y}_{S}\right) d \boldsymbol{x}=\boldsymbol{x}_{S}+i \boldsymbol{y}_{S}=\boldsymbol{z}_{S} .
$$

Equation $(78)$ is a natural extension to $\mathbb{C}^{3}$ of the formula

$$
\int_{\mathbb{R}^{3}} \boldsymbol{x} \delta\left(\boldsymbol{x}-\boldsymbol{x}_{S}\right) d \boldsymbol{x}=\boldsymbol{x}_{S}, \quad \boldsymbol{x}_{S} \in \mathbb{R}^{3} .
$$

Proposition 1 sheds some light on the nature of the source distribution $\tilde{\delta}$. 
- $L_{0}$ is the mean of $f$ over the $\operatorname{rim} \mathcal{B}$. Since $L_{1}=L_{2}=0$ when $f(\boldsymbol{x}) \equiv 1$, we see that the "charge" $Q$ resides entirely on this rim.

- $L_{1}$ is an integral of $f$ over $E_{0}$ which does not involve its derivatives, thus representing a single layer distribution on $E_{0}$. Actually, since the contributions from the rim are subtracted, the single layer resides on the interior of $E_{0}$.

- $L_{2}$ is an integral of the normal derivative of $f$ over $E_{0}$, so it represents a double layer distribution which may be regarded as equal and opposite charge distributions on $E_{0}^{+}$and $E_{0}^{-}$. This is confirmed by $\boldsymbol{P}=-i \boldsymbol{y}$.

Proposition 5 The source distribution in $\mathbb{C}^{4}$,

$$
\tilde{\delta}(\boldsymbol{z})=-\Delta \frac{1}{4 \pi^{2} \gamma^{2}},
$$

acts on a test function $f \in C^{1}\left(\mathbb{R}^{4}\right)$ in cylindrical coordinates as follows:

$$
\langle\tilde{\delta}, f\rangle=\bar{f}(a, 0)+a \bar{f}_{\rho}(a, 0)-i a \bar{f}_{\zeta}(a, 0) .
$$

Proof: From (73) we find

$$
F(\rho)=\rho\left[\bar{f}(\rho, 0)+i \frac{a^{2}-\rho^{2}}{2 a} \bar{f}_{\zeta}(\rho, 0)\right],
$$

and (72) becomes $\langle\tilde{\delta}, f\rangle=\partial_{\rho} F(a)$, which gives (79).

As $a \rightarrow 0$, this gives $\langle\tilde{\delta}, f\rangle \rightarrow f(\mathbf{0})$, which confirms that $\tilde{\delta}(\boldsymbol{x}+i \boldsymbol{y}) \rightarrow \delta(\boldsymbol{x})$. This property and $Q=1, \boldsymbol{P}=$ $-i \boldsymbol{y}, \boldsymbol{C}\left(\boldsymbol{z}_{S}\right)=\boldsymbol{z}_{S}$, as in Proposition $\mathbb{\theta}$, can be shown to hold for all $n$ [K00].

The holomorphic potential in $\mathbb{C}^{4}$ was derived from a different point of view in [K94, Section 11.2], where it was shown to decompose into causal (retarded) and anticausal (advanced) "physical wavelets." These are closely related to the complex-source pulsed beams in the engineering literature [HF89].

\section{Connection to Spacetime and Wave Equations}

It was proposed in Section 1 that point sources in physics be replaced by extended sources based on a continuation of physics to complex spacetime. The distribution $\tilde{\delta}(\boldsymbol{z})$ in complex space $\left(\mathbb{C}^{n}\right)$ seems like a promising model, since it is supported on the disk $E_{0}$ (when $n$ is odd) or the sphere $\mathcal{B}$ (when $n$ is even). But to do physics we need to add time to this complex-space formalism. We will first give an argument suggesting that time is already included in $\mathbb{C}^{n+1}$, then justfy this by proving that with this interpretation, the extended source distribution $\tilde{\delta}(\mathbf{z})$ in $\mathbb{C}^{n+1}$ acts as a propagator in spacetime, generating solutions of the Cauchy problem for the wave equation from their initial values.

Let us write

$$
\begin{array}{lll}
\mathbf{z}=\mathbf{x}+i \mathbf{y}=(\boldsymbol{z}, s+i t) \in \mathbb{C}^{n+1}, & & \boldsymbol{z} \in \mathbb{C}^{n} \\
\mathbf{x}=(\boldsymbol{x}, s), \quad \mathbf{y}=(\boldsymbol{y}, t) \in \mathbb{R}^{n+1}, & & |\boldsymbol{x}|=r .
\end{array}
$$

Analytic potential theory in $\mathbb{C}^{n+1}$ is based on the complex distance function $\gamma(\mathbf{z})$ defined by

$$
\gamma^{2} \equiv \mathbf{z}^{2}=\boldsymbol{z}^{2}+(s+i t)^{2}, \quad \operatorname{Re} \gamma \geq 0,
$$

which reduces to the Euclidean metric in $\mathbb{R}^{n+1}$ when $\mathbf{y} \rightarrow \mathbf{0}$,

$$
\gamma^{2} \rightarrow \mathbf{x}^{2}=r^{2}+s^{2},
$$


and to the Lorentzian metric in $\mathbb{R}^{n, 1}$ when $s \rightarrow 0$ and $\boldsymbol{y} \rightarrow \mathbf{0}$,

$$
\gamma^{2} \rightarrow r^{2}-t^{2}
$$

This suggests that the imaginary part $t$ of the complex space coordinate $z_{n+1}=s+i t$ should be interpreted as time. For this reason, we refer to its real part $s$ as the Euclidean time.

The idea of time as an imaginary space coordinate dates back to Minkowski [M23], who realized in 1908 that Einstein's new special relativity theory can be based on a unified four-dimensional spacetime. More recently, complex spacetime has become an important concept in quantum field theory [SW64, GJ87, twistor theory [PR86] and string theory [P98]. Even so, it is generally regarded as a useful mathematical tool rather than a fundamental aspect of physical reality. For example, see the discussion in [MTW73, p. 51].

In previous work K77, K78, K87, K90, K94, I have attempted to make complex spacetime "concrete" by giving detailed physical interpretations of the imaginary as well as the real coordinates. This is also the present motivation for developing holomorphic potential theory, where the gemetrical and physical significance of $\boldsymbol{y}$ has been emphasized. (The physical significance of $\boldsymbol{y}$ is related to the directivity of the physical wavelets associated with $\phi(\boldsymbol{x}+i \boldsymbol{y})$ HF89, K94, K00.)

Assume for simplicity that the Euclidean world is completely democratic with respect to the real space variables $\boldsymbol{x} \in \mathbb{R}^{n}$ and the Euclidean time $s$, so that there is no prefered direction in $\mathbb{R}^{n+1}$. Then if $\mathbf{y} \neq \mathbf{0}$, we may choose a coordinate system in which

$$
\mathbf{y}=(\mathbf{0}, t), \quad t=|\mathbf{y}|>0, \quad \mathbf{z}=(\boldsymbol{x}, s+i t) \in \mathbb{C}^{n+1} .
$$

In that case, the cylindrical coordinates in $\mathbb{R}^{n+1}$ are

$$
\zeta=\mathbf{x} \cdot \hat{\mathbf{y}}=s, \quad \rho=\sqrt{\mathbf{x}^{2}-\zeta^{2}}=r,
$$

and a vector $\mathbf{x} \in \mathbb{R}^{n+1}$ is represented by

$$
\mathbf{x}=r \boldsymbol{\sigma}+s \hat{\mathbf{y}}, \quad \boldsymbol{\sigma} \in S^{n-1} \subset \mathbb{R}^{n},
$$

where we do not distinguish between $\boldsymbol{\sigma} \in \mathbb{R}^{n}$ and $(\boldsymbol{\sigma}, 0) \in \mathbb{R}^{n+1}$. As before, we denote by $d \boldsymbol{\sigma}$ the normalized surface measure on $S^{n-1}$, so that $\int d \sigma=1$ and

$$
\bar{f}(r, s) \equiv \int_{S^{n-1}} f(r \boldsymbol{\sigma}+s \hat{\mathbf{y}}) d \boldsymbol{\sigma}
$$

is the mean of $f(\boldsymbol{x}, s)$ over the sphere $|\boldsymbol{x}|=r$.

For simplicity, we assume to begin with that the dimension $n$ of space is odd, so that $n+1$ is even and (72) can be applied to $\tilde{\delta}(\mathbf{z})$. We will later extend our results to even values of $n$ by applying (71). Using (72) with the substitutions

$$
n \rightarrow n+1 \equiv 2 k+2, \quad \rho \rightarrow r, \quad \zeta \rightarrow s, \quad a \rightarrow t
$$

gives the action of $\tilde{\delta}(\mathbf{x}+i \mathbf{y})$ on a test function $f(\mathbf{x})$ :

$$
\langle\tilde{\delta}, f\rangle=\left.\frac{t \sqrt{\pi}}{\Gamma\left(k+\frac{1}{2}\right)} D_{r}^{k} F(r)\right|_{r=t},
$$

where

$$
D_{r}=\frac{1}{2 r} \frac{\partial}{\partial r}, \quad F(r)=r^{2 k-1}\left[\bar{f}(r, 0)+i \frac{t^{2}-r^{2}}{2 k t} \bar{f}_{s}(r, 0)\right] .
$$

To establish a connection with the wave equation, define the function $\tilde{f}(\mathbf{z})$ on $\mathbb{C}^{n+1}$ by the convolution

$$
\tilde{f}(\mathbf{z}) \equiv \int_{\mathbb{R}^{n+1}} \tilde{\delta}\left(\mathbf{z}-\mathbf{x}^{\prime}\right) f\left(\mathbf{x}^{\prime}\right) d \mathbf{x}^{\prime} .
$$


This is an extension of $f$ from $\mathbb{R}^{n+1}$ to $\mathbb{C}^{n+1}$, since

$$
\mathbf{y} \rightarrow \mathbf{0} \Rightarrow \tilde{\delta}\left(\mathbf{x}+i \mathbf{y}-\mathbf{x}^{\prime}\right) \rightarrow \delta\left(\mathbf{x}-\mathbf{x}^{\prime}\right) \Rightarrow \tilde{f}(\mathbf{x}+i \mathbf{y}) \rightarrow f(\mathbf{x}) .
$$

Now

$$
\gamma(-\mathbf{z})=\gamma(\mathbf{z}) \Rightarrow \phi(-\mathbf{z})=\phi(\mathbf{z}) \Rightarrow \tilde{\delta}(-\mathbf{z})=\tilde{\delta}(\mathbf{z})
$$

hence

$$
\tilde{f}(\mathbf{x}+i \mathbf{y})=\int_{\mathbb{R}^{n+1}} \tilde{\delta}\left(\mathbf{x}^{\prime}-\mathbf{x}-i \mathbf{y}\right) f\left(\mathbf{x}^{\prime}\right) d \mathbf{x}^{\prime}=\int_{\mathbb{R}^{n+1}} \tilde{\delta}\left(\mathbf{x}^{\prime}-i \mathbf{y}\right) f^{\mathbf{x}}\left(\mathbf{x}^{\prime}\right) d \mathbf{x}^{\prime}=\left\langle\tilde{\delta}, f^{\mathbf{x}}\right\rangle,
$$

where $f^{\mathrm{x}}$ is the test function defined by

$$
f^{\mathbf{x}}\left(\mathbf{x}^{\prime}\right)=f\left(\mathbf{x}+\mathbf{x}^{\prime}\right)=f\left(\boldsymbol{x}+\boldsymbol{x}^{\prime}, s+s^{\prime}\right) .
$$

Note that in (85), it is $\tilde{\delta}\left(\mathbf{x}^{\prime}-i \mathbf{y}\right)$ rather than $\tilde{\delta}\left(\mathbf{x}^{\prime}+i \mathbf{y}\right)$ that acts on $f^{\mathbf{x}}$. We will account for this in Equation (86) below by letting $i \rightarrow-i$ in (82). Thus, since $\mathbf{z}=\mathbf{x}+i \mathbf{y}=(\boldsymbol{x}, s+i t)$, (81) gives

$$
\tilde{f}(\boldsymbol{x}, s+i t)=\left.\frac{t \sqrt{\pi}}{\Gamma\left(k+\frac{1}{2}\right)} D_{r}^{k} F^{\mathbf{x}}(r)\right|_{r=t}, \quad F^{\mathbf{x}}(r)=r^{2 k-1}\left[\bar{f}^{\mathbf{x}}(r, 0)+i \frac{r^{2}-t^{2}}{2 k t} \bar{f}_{s^{\prime}}^{\mathbf{x}}(r, 0)\right]
$$

with

$$
\bar{f}^{\mathbf{x}}\left(r, s^{\prime}\right) \equiv \int_{S^{n-1}} f^{\mathbf{x}}\left(r \boldsymbol{\sigma}+s^{\prime} \hat{\mathbf{y}}\right) d \boldsymbol{\sigma}=\int_{S^{n-1}} f\left(\mathbf{x}+r \boldsymbol{\sigma}+s^{\prime} \hat{\mathbf{y}}\right) d \boldsymbol{\sigma}=\int_{S^{n-1}} f\left(\boldsymbol{x}+r \boldsymbol{\sigma}, s+s^{\prime}\right) d \boldsymbol{\sigma} .
$$

Therefore

$$
\bar{f}^{\mathbf{x}}(r, 0)=\int_{S^{n-1}} f(\boldsymbol{x}+r \boldsymbol{\sigma}, s) d \boldsymbol{\sigma}
$$

is the mean of $f$ over the sphere of radius $r$ centered at $\mathbf{x}$ and orthogonal to $\mathbf{y}$, and

$$
\left.\bar{f}_{s^{\prime}}^{\mathbf{x}}(r, 0) \equiv \partial_{s^{\prime}} \bar{f}^{\mathbf{x}}\left(r, s^{\prime}\right)\right|_{s^{\prime}=0}=\int_{S^{n-1}} f_{s}(\boldsymbol{x}+r \boldsymbol{\sigma}, s) d \boldsymbol{\sigma} \equiv \bar{f}_{s}^{\mathbf{x}}(r, 0)
$$

is the mean of $f_{s}$ over the same sphere.

Theorem 5 Let $n=2 k+1 \geq 3$ and $f \in C^{k+2}\left(\mathbb{R}^{n+1}\right)$. Then $\tilde{f}(\boldsymbol{x}, s+i t)$ belongs to $C^{2}\left(\mathbb{R}^{n, 1}\right)$ as a function of $(\boldsymbol{x}, t)$, and it is the unique "classical" solution to the following Cauchy problem for the wave equation:

$$
\begin{gathered}
\partial_{t}^{2} \tilde{f}(\boldsymbol{x}, s+i t)=\Delta_{\boldsymbol{x}} \tilde{f}(\boldsymbol{x}, s+i t) \\
\lim _{t \rightarrow 0} \tilde{f}(\boldsymbol{x}, s+i t)=f(\boldsymbol{x}, s), \quad \lim _{t \rightarrow 0} \partial_{t} \tilde{f}(\boldsymbol{x}, s+i t)=i f_{s}(\boldsymbol{x}, s) .
\end{gathered}
$$

Furthermore, if $f(\boldsymbol{x}, s)$ can be continued analytically in $s$, then $\tilde{f}(\boldsymbol{x}, s+i t)$ is that continuation.

Proof: The first of the initial conditions is just the extension property (84), which has already been established. In view of this, the second initial condition can be written as

$$
\lim _{t \rightarrow 0}\left(\partial_{s}+i \partial_{t}\right) \tilde{f}(\boldsymbol{x}, s+i t)=0,
$$

which is the Cauchy-Riemann equation at $t=0$. If (90) were to hold in a complex neighborhood $U$ of $s_{0}$, it would imply that $\tilde{f}(\boldsymbol{x}, s+i t)$ is holomorphic in $s+i t \in U$. Since analytic continuation is unique when it exists, this proves our claim that $\tilde{f}(\boldsymbol{x}, s+i t)$ is the analytic continuation of $f(\boldsymbol{x}, s)$ whenever the latter exists. 
Without assuming analyticity, we now prove that $\tilde{f}(\boldsymbol{x}, s+i t)$ solves the above Cauchy problem. Let

$$
u(\boldsymbol{x}, t)=\tilde{f}(\boldsymbol{x}, s+i t), \quad v(\boldsymbol{x}, r)=\bar{f}^{\mathbf{x}}(r, 0), \quad w(\boldsymbol{x}, r)=i \bar{f}_{s}^{\mathbf{x}}(r, 0),
$$

where the dependence on the parameter $s$ is suppressed. Then (86) becomes

$$
u(\boldsymbol{x}, t)=\frac{t \sqrt{\pi}}{\Gamma\left(k+\frac{1}{2}\right)} D_{r}^{k}\left[r^{2 k-1} v(\boldsymbol{x}, r)+\frac{r^{2}-t^{2}}{2 k t} r^{2 k-1} w(\boldsymbol{x}, r)\right]_{r=t} .
$$

According to (87), $v(\boldsymbol{x}, r)$ is the mean of $f$ over the sphere of radius $r$ centered at $\boldsymbol{x}$, hence $v(\boldsymbol{x}, 0)=f(\boldsymbol{x}, s)$. Similarly, (88) states that $w(\boldsymbol{x}, r)$ is the mean of $i f_{s}$ over the same sphere, so $w(\boldsymbol{x}, 0)=i f_{s}(\boldsymbol{x}, s)$. We therefore need to show that $u$ solves the following Cauchy problem:

$$
\begin{gathered}
u_{t t}(\boldsymbol{x}, t)=\Delta u(\boldsymbol{x}, t) \\
u(\boldsymbol{x}, 0)=v(\boldsymbol{x}, 0), \quad u_{t}(\boldsymbol{x}, 0)=w(\boldsymbol{x}, 0) .
\end{gathered}
$$

By the definition of $D_{r}$, the left equation in (86) is

$$
\frac{\sqrt{\pi}}{2^{k} \Gamma\left(k+\frac{1}{2}\right)} \partial_{t}\left(\frac{\partial}{t \partial t}\right)^{k-1} t^{2 k-1} v(\boldsymbol{x}, t)=c_{n} \partial_{t}\left(\frac{\partial}{t \partial t}\right)^{k-1} t^{2 k-1} v(\boldsymbol{x}, t)
$$

where

$$
c_{n}=\frac{1}{1 \cdot 3 \cdots(2 k-1)=1 \cdot 3 \cdots(n-2)} .
$$

The right equation in 86 is

$$
c_{n}\left(\frac{\partial}{r \partial r}\right)^{k}\left[\frac{r^{2}-t^{2}}{2 k} r^{2 k-1} w(\boldsymbol{x}, r)\right]_{r=t} .
$$

Letting $\xi=r^{2} / 2$, a straightforward computation shows that for any function $G(\xi)$,

$$
\begin{aligned}
\left(\frac{\partial}{r \partial r}\right)^{k}\left[\left(r^{2}-t^{2}\right) G(\xi)\right] & =\partial_{\xi}^{k}\left[\left(2 \xi-t^{2}\right) G(\xi)\right]=2 k \partial_{\xi}^{k-1} G(\xi)+\left(2 \xi-t^{2}\right) \partial_{\xi}^{k} G(\xi) \\
& =2 k\left(\frac{\partial}{r \partial r}\right)^{k-1} G+\left(r^{2}-t^{2}\right)\left(\frac{\partial}{r \partial r}\right)^{k} G .
\end{aligned}
$$

Thus (95) becomes

$$
c_{n}\left(\frac{\partial}{r \partial r}\right)^{k-1}\left[r^{2 k-1} w(\boldsymbol{x}, r)\right]_{r=t}=c_{n}\left(\frac{\partial}{t \partial t}\right)^{k-1} t^{2 k-1} w(\boldsymbol{x}, t) .
$$

The sum of (94) and (96) is precisely the solution $u(\boldsymbol{x}, t)$ of the initial-value problem (93) with $n=2 k+1$, as expressed in terms of spherical means. See John [J55, Courant and Hilbert [CH62, pp. 699-703] or Folland [F95, p. 170].

That $u \in C^{2}\left(\mathbb{R}^{n, 1}\right)$ follows because $v(\boldsymbol{x}, r) \in C^{k+2}\left(\mathbb{R}^{n}\right)$ in $\boldsymbol{x}$ and (94) contains $k$ derivatives, while $w(\boldsymbol{x}, r) \in$ $C^{k+1}\left(\mathbb{R}^{n}\right)$ in $\boldsymbol{x}$ and $(96)$ contains $k-1$ derivatives. Finally, uniqueness of the solution $u$ is a general property of the Cauchy problem.

As mentioned earlier, the above result can be extended to an even number $n$ of space dimensions by applying the recursion relation $(70)$ between the singular source distributions in $\mathbb{C}^{n}$ and $\mathbb{C}^{n+1}$. In terms of the solutions $\tilde{f}(\boldsymbol{x}, s+i t)$ to the Cauchy problem, this amounts to using Hadamard's method of descent $\mathrm{H} 52$, CH62. Consequently, the same formula (85) gives the solution of the Cauchy problem for the wave equation in $\mathbb{R}^{n, 1}$ for all values of $n \geq 2$. 
The support properties of $\tilde{\delta}$ now imply some important attributes of waves in $\mathbb{R}^{n, 1}$. For $\mathbf{z}=\mathbf{x}+i \mathbf{y} \in \mathbb{C}^{n+1}$ with $\mathbf{y} \neq \mathbf{0}$, recall that

$$
\operatorname{supp} \tilde{\delta}(\mathbf{x}+i \mathbf{y})= \begin{cases}\mathcal{B}(\mathbf{y}) & \text { for odd } n \geq 3 \\ E_{0}(\mathbf{y}) & \text { for all other } n \geq 2 .\end{cases}
$$

From (85) we can therefore immediately draw the following conclusions about waves (solutions of the wave equation) $u(\boldsymbol{x}, t)$ in $n$ space dimensions:

- For odd $n \geq 3, u(\boldsymbol{x}, t)$ depends on the values of $u(\boldsymbol{x}+\boldsymbol{v}, 0)$ and $u_{t}(\boldsymbol{x}+\boldsymbol{v}, 0)$ only in an arbitrarily thin shell containing the sphere $|\boldsymbol{v}|=t$. (We need a shell, rather than the sphere itself, because of the derivatives appearing in (94) and (96).) This is the strong form of Huygens' principle [BC87], which states that $u$ depends on the initial data only on the light cone.

- For all other $n \geq 2, u(\boldsymbol{x}, t)$ depends on the values of $u(\boldsymbol{x}+\boldsymbol{v}, 0)$ and $u_{t}(\boldsymbol{x}+\boldsymbol{v}, 0)$ in the past cone $|\boldsymbol{v}| \leq t$. This is the principle of causality, which states that no signal (information, energy) can travel with speed greater than $c=1$. (If we rescale time by $t \rightarrow c t$ with arbitrary $c>0$, then the maximum propagation speed is $c$.)

We emphasize that although holomorphy was needed in the definition of $\tilde{\delta}$, it is not necessary for the above relation to the wave equation. This distinguishes our results from all similar results in the literature of which I am aware, where holomorphy of the Cauchy data is essential. See Garabedian [G64, pp. 191-202] and Ryan R90, R90a, R96a.

Theorem and its counterpart for even $n$ can be extended in various ways.

- Clearly it is not necessary to assume that $t>0$, since the support of $\tilde{\delta}(\mathbf{x}+i \mathbf{y})$ is symmetric with respect to $\mathbf{y} \rightarrow-\mathbf{y}$. When $t<0, \tilde{f}(\boldsymbol{x}, s+i t)$ is a solution of the "final-value problem" in terms of the Cauchy data at $t=0$.

- The Cauchy data $f(\mathbf{x})$ need not belong to $C^{k+2}\left(\mathbb{R}^{n+1}\right)$. When $f$ is a distribution belonging, say, to some Sobolev space [F95, Chapter 6], then $\tilde{f}(\boldsymbol{x}, s+i t)$ is a distributional solution and the derivatives in (94) and (96) must be interpreted as distributional derivatives.

- To solve the inhomogeneous wave equation

$$
\begin{gathered}
u_{t t}(\boldsymbol{x}, t)-\Delta u(\boldsymbol{x}, t)=j(\boldsymbol{x}, t) \\
u(\boldsymbol{x}, 0)=v(\boldsymbol{x}), \quad u_{t}(\boldsymbol{x}, 0)=w(\boldsymbol{x}),
\end{gathered}
$$

one can apply Duhamel's principle to solutions of the homogeneous equation [F95]. This involves integration of $\tilde{\delta}\left(\mathbf{z}^{\prime}\right)$ on the truncated solid light cone with $0 \leq t^{\prime} \leq t$. However, we will see that in the Clifford setting, the current formalism leads directly to solutions of inhomogeneous hyperbolic equations, where the time-dependent source is determined by the given function $f(\mathbf{x})$ in Euclidean spacetime.

\section{Extension to Clifford Analysis}

Clifford and quaternionic analyses BDS82, R96, GS97, R98] are generalizations to $\mathbb{R}^{n}$ of one-dimensional complex analysis that are proving to be a unifying and very powerful tool in physics H66, KS96, MM98, O98, B99. We now show that all the above constructions generalize naturally to this setting. Let $C \ell_{n}$ be the complex Clifford algebra generated by elements $e_{1}, \cdots, e_{n}$ satisfying the anticommutation relations

$$
e_{k} e_{l}+e_{l} e_{k}=2 \delta_{k l} \quad 1 \leq k, l \leq n .
$$


As a complex vector space, $C \ell_{n}$ has dimension $2^{n}$ with basis vectors

$$
e_{K} \equiv e_{k_{1}} \cdots e_{k_{p}}, \quad K=\left\{k_{1}, \cdots, k_{p}\right\}, \quad 0 \leq p \leq n, \quad 1 \leq k_{1}<\cdots<k_{p} \leq n
$$

where the element labeled by the empty set $K=\varnothing(p=0)$ is by definition $e_{\varnothing}=1$. Thus a general vector has the form $v=\sum_{K} c_{K} e_{K}$, where $c_{K} \in \mathbb{C}$ and the sum runs over the $2^{n}$ sets $K$ as above. The element $\sum_{k=1}^{n} z_{k} e_{k} \in C \ell_{n}$ is identified with the vector $\boldsymbol{z} \in \mathbb{C}^{n}$, and by (97) the products and squares of such vectors satisfy

$$
\boldsymbol{z} \boldsymbol{w}+\boldsymbol{w} \boldsymbol{z}=2 \sum_{k=1}^{n} z_{k} w_{k} \equiv 2 \boldsymbol{z} \cdot \boldsymbol{w}, \quad \boldsymbol{z}^{2}=\boldsymbol{z} \cdot \boldsymbol{z} \equiv \gamma(\boldsymbol{z})^{2}
$$

where $\gamma(\boldsymbol{z})$ is the complex Euclidean distance function. This connection will be our basis for generalizing holomorphic potential theory to the Clifford setting.

Clifford analysis is a noncommutative calculus dealing with Clifford-valued functions

$$
f: \mathbb{R}^{n} \rightarrow C \ell_{n}, \quad f(\boldsymbol{x})=\sum_{K} e_{K} f_{K}(\boldsymbol{x}) \quad \text { where } \quad f_{K}: \mathbb{R}^{n} \rightarrow \mathbb{C} .
$$

The primary tool is the Dirac operator $\boldsymbol{D}=\sum_{k} e_{k} \partial_{k}$, which is closely related to the exterior derivative AM78] but in addition incorporates the underlying metric. It acts on Clifford-valued functions from either left or right by

$$
\begin{gathered}
\boldsymbol{D} f \equiv \sum_{k=1}^{n} e_{k} \frac{\partial f}{\partial x_{k}} \neq f \overleftarrow{\boldsymbol{D}} \equiv \sum_{k=1}^{n} \frac{\partial f}{\partial x_{k}} e_{k} \\
\Rightarrow \boldsymbol{D}^{2} f=f \overleftarrow{\boldsymbol{D}}^{2}=\sum_{K} e_{K} \Delta f_{K}=\Delta f
\end{gathered}
$$

Thus $\boldsymbol{D}$ is a "square root" of the Laplacian $\Delta$ in $\mathbb{R}^{n}$. It is an elliptic operator because the relations (97) are based on the Euclidean metric in $\mathbb{R}^{n}$. In 1928, Dirac formulated a similar operator in the Minkowskian spacetime $\mathbb{R}^{3,1}$, where it is hyperbolic and its square is the wave operator. This formed the basis for his relativistic wave equation of the electron [D58], which had a revolutionary impact on physics, including especially the dramatic prediction of antimatter. Mathematicians usually prefer Euclidean Dirac operators because, among other things, they yield powerful methods for solving boundary-value problems generalizing those in one-dimensional complex analysis GS97. For this and similar reasons, most mathematical work on Dirac operators is restricted to the elliptic case; see the discussion in 098 , p. 1]. Consequently, rigorous analyses of Maxwell's equations by Clifford methods usually deal with static or time-harmonic fields KS96, MM98]. By assuming that the boundary/Cauchy data is holomorphic, it is possible to arrive at solutions of hyperbolic Dirac equations through analytic continuation R90, R90a, R96a, generalizing the method employed by Garabedian for establishing a connection between solutions of the Laplace and wave equations G64. As in Section 6, the present method is not restricted by this assumption. We will "Cliffordize" the holomorphic potential theories in $\mathbb{C}^{n}$ and $\mathbb{C}^{n+1}$, interpreted respectively as complex space and complex spacetime. The relation established in Section 6 between Laplacians and wave operators then yields the desired connection between elliptic and hyperbolic Dirac operators.

The Clifford counterpart of the Newtonian potential $\phi(\boldsymbol{x})$ is the Cauchy kernel $C: \mathbb{R}^{n} \rightarrow C \ell_{n}$, defined as the fundamental solution of $\boldsymbol{D}$ :

$$
\boldsymbol{D} C(\boldsymbol{x})=C(\boldsymbol{x}) \stackrel{\leftarrow}{\boldsymbol{D}}=\delta(\boldsymbol{x}), \quad \lim _{r \rightarrow \infty} C(\boldsymbol{x})=0
$$

Because $\boldsymbol{D}^{2}=\Delta$, the solution is easily expressed in terms of the Newtonian potential:

$$
\phi=\frac{r^{2-n}}{\omega_{n}(2-n)}, \quad C(\boldsymbol{x})=\boldsymbol{D} \phi(\boldsymbol{x})=\frac{\boldsymbol{x}}{\omega_{n} r^{n}} .
$$


The expression on the right remains valid for $n=2$ if we take $\phi=(2 \pi)^{-1} \ln r$. Replacing $r(\boldsymbol{x})$ with $\gamma(\boldsymbol{z})$ immediately gives an extension to $\mathbb{C}^{n}$, where the point source $\delta$ becomes the extended source $\tilde{\delta}$ :

$$
\begin{aligned}
C(\boldsymbol{z}) & \equiv \boldsymbol{D} \phi(\boldsymbol{z})=\phi(\boldsymbol{z}) \overleftarrow{\boldsymbol{D}}=\frac{\boldsymbol{z}}{\omega_{n} \gamma^{n}}, \quad \boldsymbol{z} \in \mathbb{C}^{n}, n \geq 2 \\
& \Rightarrow \quad \boldsymbol{D} C(\boldsymbol{z})=C(\boldsymbol{z}) \overleftarrow{\boldsymbol{D}}=\Delta \phi(\boldsymbol{z})=\tilde{\delta}(\boldsymbol{z}) .
\end{aligned}
$$

For all even $n \geq 2, C(\boldsymbol{z})$ is holomorphic on the complement of the null cone $\mathcal{N}$. For odd $n \geq 3$, it inherits the branch cut from $\gamma$. Thus for all $n \geq 2, C(\boldsymbol{z})$ is holomorphic on the complement of the set

$$
\mathcal{S}_{n}= \begin{cases}\left\{\boldsymbol{x}+i \boldsymbol{y} \mid(\boldsymbol{x}+i \boldsymbol{y})^{2}=0\right\} & \text { for even } n \geq 2 \\ \left\{\boldsymbol{x}+i \boldsymbol{y} \in \mathbb{C}^{n} \mid \boldsymbol{x} \in E_{0}(\boldsymbol{y})\right\} & \text { for odd } n \geq 3\end{cases}
$$

Given a Clifford-valued test function $f: \mathbb{R}^{n} \rightarrow C \ell_{n}$, we define its extension to $\tilde{f}: \mathbb{C}^{n} \rightarrow C \ell_{n}$ exactly as before:

$$
\tilde{f}(\boldsymbol{z}) \equiv \int_{\mathbb{R}^{n}} \tilde{\delta}\left(\boldsymbol{x}^{\prime}-\boldsymbol{z}\right) f\left(\boldsymbol{x}^{\prime}\right) d \boldsymbol{x}^{\prime}, \Rightarrow \lim _{\boldsymbol{y} \rightarrow \mathbf{0}} \tilde{f}(\boldsymbol{x}+i \boldsymbol{y})=f(\boldsymbol{x}) .
$$

Recall that for $\tilde{f}$ to be defined, $f$ must be $C^{k}$ with $k=\left[\frac{n-1}{2}\right]$ if $n \geq 3$. It can also be shown K00] that $f$ must be $C^{1}$ if $n=2$. Inserting one of the expressions (103) for $\tilde{\delta}$ and integrating by parts gives

$$
\tilde{f}(\boldsymbol{z})=\int_{\mathbb{R}^{n}} C\left(\boldsymbol{x}^{\prime}-\boldsymbol{z}\right) \overleftarrow{\boldsymbol{D}} f\left(\boldsymbol{x}^{\prime}\right) d \boldsymbol{x}^{\prime}=-\int_{\mathbb{R}^{n}} C\left(\boldsymbol{x}^{\prime}-\boldsymbol{z}\right) \boldsymbol{D} f\left(\boldsymbol{x}^{\prime}\right) d \boldsymbol{x}^{\prime}
$$

where $\overleftarrow{\boldsymbol{D}}$ denotes the left-acting Dirac opeator with respect to $\boldsymbol{x}^{\prime}$. We will use this expression to derive an extended version of the Borel-Pompeiu formula. Let $M$ be a bounded domain in $\mathbb{R}^{n}$ with piecewise smooth $\left(C^{1}\right)$ boundary $\partial M$, and let

$$
f_{M}(\boldsymbol{x})=\chi_{M}(\boldsymbol{x}) f(\boldsymbol{x}), \quad \text { where } \quad \chi_{M}(\boldsymbol{x})= \begin{cases}1 & \text { if } \boldsymbol{x} \in M \\ 0 & \text { if } \boldsymbol{x} \in \bar{M}^{\prime} \equiv \mathbb{R}^{n} \backslash \bar{M} .\end{cases}
$$

We do not need to define $f_{M}$ on $\partial M$. Taking the distributional derivatives of $f_{M}$ gives

$$
\boldsymbol{D} f_{M}(\boldsymbol{x})=\left(\boldsymbol{D} \chi_{M}(\boldsymbol{x})\right) f(\boldsymbol{x})+\chi_{M}(\boldsymbol{x}) \boldsymbol{D} f(\boldsymbol{x}) .
$$

We want to substitute this into (105) to obtain an expression for the extension $\tilde{f}_{M}(\boldsymbol{z})$ of $f_{M}(\boldsymbol{x})$. This will make sense if the singularities of $\overline{\boldsymbol{D} f_{M}}$ do not meet the singularities of $C(\boldsymbol{x}-\boldsymbol{z})$. That will be the case if we assume that

$$
\boldsymbol{z} \notin \partial M+\mathcal{S}_{n} \equiv\left\{\boldsymbol{x}_{b}+\boldsymbol{z} \mid \boldsymbol{x}_{b} \in \partial M, \boldsymbol{z} \in \mathcal{S}_{n}\right\}
$$

and we refer to such points $\boldsymbol{z} \in \mathbb{C}^{n}$ as regular with respect to $\partial M$. Note that when $\boldsymbol{z} \in \mathbb{R}^{n}$, this means simply that $\boldsymbol{z} \notin \partial M$. For $\boldsymbol{z}$ regular, we may substitute (106) into (105) to obtain

$$
\tilde{f}_{M}(\boldsymbol{z})=-\int_{\mathbb{R}^{n}} C\left(\boldsymbol{x}^{\prime}-\boldsymbol{z}\right)\left(\boldsymbol{D} \chi_{M}\left(\boldsymbol{x}^{\prime}\right)\right) f\left(\boldsymbol{x}^{\prime}\right) d \boldsymbol{x}^{\prime}-\int_{\mathbb{R}^{n}} C\left(\boldsymbol{x}^{\prime}-\boldsymbol{z}\right) \chi_{M}\left(\boldsymbol{x}^{\prime}\right) \boldsymbol{D} f\left(\boldsymbol{x}^{\prime}\right) d \boldsymbol{x}^{\prime} .
$$

We claim that the first term can be written in classical (non-distributional) form as

$$
\int_{\partial M} C\left(\boldsymbol{x}^{\prime}-\boldsymbol{z}\right) \boldsymbol{n}\left(\boldsymbol{x}^{\prime}\right) f\left(\boldsymbol{x}^{\prime}\right) d \sigma\left(\boldsymbol{x}^{\prime}\right)
$$

where $\boldsymbol{n}(\boldsymbol{x})$ is the outgoing unit normal at $\boldsymbol{x} \in \partial M$ and $d \sigma(\boldsymbol{x})$ is the area measure on $\partial M$ induced from the volume measure $d \boldsymbol{x}$. To see this, note that there exists a differentiable function $\mu(\boldsymbol{x})$ such that

$$
M=\{\boldsymbol{x} \mid \mu(\boldsymbol{x})>0\}, \quad \partial M=\{\boldsymbol{x} \mid \mu(\boldsymbol{x})=0\}, \quad \nabla \mu(\boldsymbol{x})=-|\nabla \mu(\boldsymbol{x})| \boldsymbol{n}(\boldsymbol{x}) .
$$


Then $\chi_{M}$ can be expressed in terms of the Heaviside step function $\theta$ by $\chi_{M}(\boldsymbol{x})=\theta(\mu(\boldsymbol{x}))$, hence

$$
\boldsymbol{D} \chi_{M}(\boldsymbol{x})=\delta(\mu(\boldsymbol{x})) \boldsymbol{D} \mu(\boldsymbol{x})=\delta(\mu(\boldsymbol{x})) \nabla \mu(\boldsymbol{x})=-\delta(\mu(\boldsymbol{x}))|\nabla \mu(\boldsymbol{x})| \boldsymbol{n}(\boldsymbol{x}) .
$$

The connection to the expression (109) can now be made by using the implicit function theorem with $\mu(\boldsymbol{x})$ as one of the local coordinates. The second term in (108) reduces to the integral over $M$, giving the following result.

\section{Theorem 6 (Extended Borel-Pompeiu formula)}

Let $\boldsymbol{z} \in \mathbb{C}^{n}$ be regular with respect to $\partial M$. Then

$$
\tilde{f}_{M}(\boldsymbol{z})=\int_{\partial M} C\left(\boldsymbol{x}^{\prime}-\boldsymbol{z}\right) \boldsymbol{n}\left(\boldsymbol{x}^{\prime}\right) f\left(\boldsymbol{x}^{\prime}\right) d \sigma\left(\boldsymbol{x}^{\prime}\right)-\int_{M} C\left(\boldsymbol{x}^{\prime}-\boldsymbol{z}\right) \boldsymbol{D} f\left(\boldsymbol{x}^{\prime}\right) d \boldsymbol{x}^{\prime} .
$$

For $\boldsymbol{z} \rightarrow \boldsymbol{x} \in \mathbb{R}^{n} \backslash \partial M$, Equation (104) applied to $f_{M}$ reproduces $f_{M}(\boldsymbol{x})$ since $\tilde{\delta}\left(\boldsymbol{x}^{\prime}-\boldsymbol{x}\right)=\delta\left(\boldsymbol{x}^{\prime}-\boldsymbol{x}\right)$. Thus (110) reduces to the usual Borel-Pompeiu formula for Clifford-valued functions GS97,

$$
\int_{\partial M} C\left(\boldsymbol{x}^{\prime}-\boldsymbol{x}\right) \boldsymbol{n}\left(\boldsymbol{x}^{\prime}\right) f\left(\boldsymbol{x}^{\prime}\right) d \sigma\left(\boldsymbol{x}^{\prime}\right)-\int_{M} C\left(\boldsymbol{x}^{\prime}-\boldsymbol{x}\right) \boldsymbol{D} f\left(\boldsymbol{x}^{\prime}\right) d \boldsymbol{x}^{\prime}= \begin{cases}f(\boldsymbol{x}), & x \in M \\ 0, & \boldsymbol{x} \in \bar{M}^{\prime} .\end{cases}
$$

We may interpret $f(\boldsymbol{x})$ as a field generated by the source function

$$
j(\boldsymbol{x}) \equiv \boldsymbol{D} f(\boldsymbol{x}) .
$$

Then (111) solves the boundary-value problem for (112), expressing the field $f$ inside $M$ in terms of its sources in $M$ and its values on $\partial M$. (To investigate the limit of (111) as $\boldsymbol{x} \rightarrow \partial M$, one also needs the Plemelj-Sokhotzki formula GS97.) In particular, $f$ is said to be left-monogenic in $M$ if $j(\boldsymbol{x})=0$ in $M$. In that case, the second term in (111) vanishes and the Borel-Pompeiu formula reduces to a multidimensional generalization of Cauchy's integral formula, with monogenicity replacing holomorphy.

To describe waves, such as a time-dependent electromagnetic field, we ascend to $\mathbb{C}^{n+1}$ as explained earlier. In the notation of Section 6 , let

$$
\begin{gathered}
\boldsymbol{z}=\boldsymbol{x}+i \boldsymbol{y} \in \mathbb{C}^{n}, \quad \mathbf{z}=\left(\boldsymbol{z}, z_{0}\right)=\mathbf{x}+i \mathbf{y} \in \mathbb{C}^{n+1} \\
\mathbf{y}=(\mathbf{0}, t) \quad \mathbf{x}=(\boldsymbol{x}, s) \Rightarrow \mathbf{z}=(\boldsymbol{x}, s+i t)
\end{gathered}
$$

The generators of $C \ell_{n+1}$ are $\left\{e_{0}, \cdots, e_{n}\right\}$, satisfying relations identical to (97) but with $0 \leq k, l \leq n$. The elliptic Dirac operators in $\mathbb{R}^{n}$ and $\mathbb{R}^{n+1}$ are

$$
\boldsymbol{D}=\sum_{k=1}^{n} e_{k} \partial_{k}, \quad \mathbf{D} \equiv \sum_{k=0}^{n} e_{k} \partial_{k}=\boldsymbol{D}+e_{0} \partial_{s}, \quad \partial_{0} \equiv \partial_{s}
$$

We also define the hyperbolic (space-time) Dirac operator by formally substituting $s \rightarrow i t$ in $\mathbf{D}$ :

$$
\tilde{\mathbf{D}} \equiv \boldsymbol{D}-i e_{0} \partial_{t} .
$$

Then

$$
\mathbf{D}^{2}=\Delta+\partial_{s}^{2} \quad \text { and } \quad \tilde{\mathbf{D}}^{2}=\Delta-\partial_{t}^{2}=\square,
$$

where $\Delta$ is the spatial Laplacian in $\mathbb{R}^{n}$ and $\square$ is the wave operator in $\mathbb{R}^{n, 1}$.

Theorem 7 (Inhomogeneous Dirac/Maxwell Equation) For $n=2 k+1 \geq 3$, let $f: \mathbb{R}^{n+1} \rightarrow C \ell_{n+1}$ be a $C^{k+2}$ function and define the functions $j: \mathbb{R}^{n+1} \rightarrow C \ell_{n+1}$ and $\tilde{j}: \mathbb{C}^{n+1} \rightarrow C \ell_{n+1}$ by

$$
\mathbf{D} f(\boldsymbol{x}, s)=j(\boldsymbol{x}, s), \quad \tilde{\mathbf{D}} \tilde{f}(\boldsymbol{x}, s+i t)=\tilde{j}(\boldsymbol{x}, s+i t) .
$$


Then $\tilde{j}(\boldsymbol{x}, s+i t)$ is a $C^{1}$ solution of

$$
\tilde{\mathbf{D}} \tilde{j}(\boldsymbol{x}, s+i t)=0,
$$

and the system $\{\tilde{f}, \tilde{j}\}$ satisfies the initial conditions

$$
\tilde{f}(\boldsymbol{x}, s)=f(\boldsymbol{x}, s), \quad \tilde{j}(\boldsymbol{x}, s)=j(\boldsymbol{x}, s) .
$$

Proof: This follows directly from Theorem 5 . Since $\tilde{f}(\boldsymbol{x}, s+i t)$ is a $C^{2}$ solution of the homogeneous wave equation, $\tilde{j}(\boldsymbol{x}, s+i t)$ is $C^{1}$ and

$$
\tilde{\mathbf{D}} \tilde{j}=\tilde{\mathbf{D}}^{2} \tilde{f}=\square \tilde{f}=0 .
$$

From the initial conditions on $\tilde{f}$, we have $\tilde{f}(\boldsymbol{x}, s)=f(\boldsymbol{x}, s)$ and

$$
\begin{aligned}
\lim _{t \rightarrow 0} \tilde{j}(\boldsymbol{x}, s+i t) & =\boldsymbol{D} \tilde{f}(\boldsymbol{x}, s)-i e_{0} \lim _{t \rightarrow 0} \partial_{t} \tilde{f}(\boldsymbol{x}, s+i t) \\
& =\boldsymbol{D} f(\boldsymbol{x}, s)+e_{0} \partial_{s} f(\boldsymbol{x}, s)=\mathbf{D} f(\boldsymbol{x}, s)=j(\boldsymbol{x}, s) .
\end{aligned}
$$

For $n=3$, the right side of $(\sqrt{113})$ is precisely the Clifford form of the inhomogeneous, time-dependent Maxwell equations [H66, 098, B99], where

$$
\tilde{f}(\boldsymbol{x}, s+i t)=\sum_{0=\mu<\nu}^{3} e_{\mu \nu} F_{\mu \nu}(\boldsymbol{x}, t) \equiv F(\boldsymbol{x}, t)
$$

is the electromagnetic field (a bivector),

$$
\tilde{j}(\boldsymbol{x}, s+i t)=\sum_{\mu=0}^{3} e_{\mu} J_{\mu}(\boldsymbol{x}, t) \equiv J(\boldsymbol{x}, t)
$$

is the charge-current density (a four-vector), and the Euclidean time $s$ is a free parameter. As with our distance function, the Minkowskian metric appears in (116) and (117) when the time components $J_{0}$ and $F_{0 k}(k=1,2,3)$ are imaginary and the spatial components are real.

Again, we did not need to assume that $\tilde{f}$ or $\tilde{j}$ are holomorphic in $s+i t$. Note that although $\tilde{j}(\boldsymbol{x}, s+i t)$ is a time-evolved extension of $j(\boldsymbol{x}, s)$, it can be shown [K00] that it is not the one obtained by convolving with $\tilde{\delta}$. Instead, the time dependence of the charge-current density is governed by (114), the scalar part of which is the continuity equation implying conservation of the total charge.

In (113) and (114), we have taken $M=\mathbb{R}^{n+1}$ for simplicity, giving a pure initial-value problem. These equations may also be formulated in a bounded region $M \subset \mathbb{R}^{n+1}$ by using the extended Borel-Pompeiu formula for $\tilde{f}_{M}(\boldsymbol{x}, s+i t)$. This suggests that Theorem 6 may be used to solve mixed initial/boundary value problems, provided one is careful about dealing with the singular points $(\boldsymbol{x}, s+i t) \in \partial M+\mathcal{S}_{n+1}$. These are in the domain of influence of the boundary; that is, they can be reached at time $t$ by signals originating from the boundary $\partial M$ at time $t=0$. However, this still does not give a mechanism for boundary effects initiated at $t>0$ (such as reflections) to influence the solution. This and related questions will be treated elsewhere.

\section{Acknowledgements}

This work was supported by AFOSR Contract \# F49620-98-C-0013. It is a pleasure to thank Arje Nachman for encouragement and Paul Garabedian, Sigurdur Helgason, David Jerison, John Ryan and Frank Sommen for stimulating and enjoyable conversations. 


\section{References}

[AM78] R Abraham and JE Marsden, Foundations of Classical Mechanics, second edition, BenjaminCummings, Reading, 1978.

[BC87] B.B. Baker and E.T. Copson, The Mathematical Theory of Huygens' Principle, Third Edition, Chelsea, New York, 1987.

[B99] WE Baylis, Electrodynamics: A Modern Geometric Approach, Birkhäuser, Boston, 1999.

[BDS82] F Brackx, R Delanghe and F Sommen, Clifford Analysis, Pitman, Boston, 1982.

[C99] TY Cao, Conceptual Foundations of Quantum Field Theory, Cambridge University Press, 1999.

[CH62] R Courant and D Hilbert, Methods of Mathematical Physics, Vol. II, Interscience, New York, 1962.

[D58] PAM Dirac, The Principles of Quantum Mechanics, Fourth Edition, Oxford, 1958.

[F64] RP Feynman, Lectures on Physics, Vol II, Addison-Wesley, Reading, 1964.

[F95] GB Folland, Introduction to Partial Differential Equations, Second Edition, Princeton University Press, 1995.

[G64] PR Garabedian, Partial Differential Equations, Chelsea, New York, 1964; AMS Chelsea, Providence, 1998.

[GS64] IM Gelfand and GE Shilov, Generalized Functions, Volume 1: Properties and Operations, Academic Press, New York, 1964.

[GJ87] J Glimm and A Jaffe, Quantum Physics: A Functional Integral Point of View, Second Edition, Springer-Verlag, New York, 1987.

[GS97] K Gürlebeck and W Sprössig, Quaternionic and Clifford Calculus for Physicists and Engineers, John Wiley \& Sons, Chichester, 1997.

[H52] J Hadamard, Lectures on Cauchy's Problem, Dover, New York, 1952.

[HF89] E Heyman and LB Felsen, Complex source pulsed beam fields, J. Optical Soc. America 6, 806-817, 1989.

[H66] D Hestenes, Space-Time Algebra, Gordon and Breach, New York, 1966.

[J99] JD Jackson, Classical Electrodynamics, third edition, John Wiley \& Sons, New York, 1999.

[J55] F. John, Plane Waves and Spherical Means, Interscience, New York, 1955.

[K77] G Kaiser, Phase-space approach to relativistic quantum mechanics, Part I: Coherent-state representation for massive scalar particles, J. Math. Phys. 18 (1977), 952-959.

[K78] G Kaiser, Phase-space approach to relativistic quantum mechanics, Part II: Geometrical aspects, J. Math. Phys. 19 (1978), 502-507.

[K80] G Kaiser, Phase-space approach to relativistic quantum mechanics, Part III: Quantization, relativity, localization and gauge freedom, J. Math. Phys. 22 (1980), 705-714.

[K87] G Kaiser, Quantized fields in complex spacetime, Annals of Physics 173 (1987), 338-354.

[K90] G Kaiser, Quantum Physics, Relativity, and Complex Spacetime, North-Holland, Amsterdam, 1990.

[K94] G Kaiser, A Friendly Guide to Wavelets, Birkhäuser, Boston, 1994.

[K00] G Kaiser, Physical Wavelets and Wave Equations, Birkhäuser, Boston, 2000, in preparation. 
[KS96] VV Kravchenko and MV Shapiro, Integral Representations for Spatial Models of Mathematical Physics, Addison Wesley Longman, Ltd., 1996, available from CRC Press.

[M23] H Minkowski, Space and Time, translated from the German and reprinted in The Principle of Relativity, edited by HA Lorentz, A Einstein, H Minkowski and H Weyl, Dover, 1923.

[MTW73] CW Misner, KS Thorne and JA Wheeler, Gravitation, WH Freeman, San Francisco, 1973.

[MM98] A McIntosh and M Mitrea, Clifford algebras and Maxwell's equations in Lipschitz domains, preprint, 1998. To appear in Mathematical Methods in Applied Sciences.

[O98] E Obolashvili, Partial Differential Equations in Clifford Analysis, Addison Wesley Longman, Ltd., 1998, available from CRC Press.

[PR86] R Penrose and W Rindler, Spinors and Space-Time, Volume 2, Cambridge University Press, 1986.

[P98] J Polchinski, String Theory, Volumes 1 and 2, Cambridge University Press, 1998.

[R90] J Ryan, Complex Clifford analysis and domains of holomorphy, J. Austral. Math. Soc. (Series A) 48 (1990), 413-433.

[R90a] J Ryan, Cells of harmonicity and generalized Cauchy integral formulae, Proc. London Math. Soc. 60 (1990), 295-318.

[R96] J Ryan (Editor), Clifford Algebras in Analysis and Related Topics, CRC, 1996.

[R96a] J Ryan, Intrinsic Dirac operators, Advances in Mathematics 118 (1996), 99-133.

[R98] J Ryan, Dirac Operators in Analysis, Addison Wesley Longman, Ltd., 1998, available from CRC Press.

[SW64] RF Streater and AS Wightman, PCT, Spin and Statistics, and All That, Addison-Wesley, 1964.

[T96] N Temme, Special Functions, John Wiley \& Sons, New York, 1996.

[T75] F Treves, Basic Linear Partial Differential Equations, Academic Press, New York, 1975.

[WF45] JA Wheeler and RP Feynman, Interaction with the absorber as the mechanism of radiation, Rev. Mod. Phys. 17 (1945), 157-181.

[WF49] JA Wheeler and RP Feynman, Classical electrodynamics in terms of direct interparticle action, Rev. Mod. Phys. 21 (1949), 425-433.

[Z65] AH Zemanian, Distribution Theory and Transform Analysis, McGraw-Hill, New York, 1965. 